Charles Pugh $\cdot$ Michael Shub

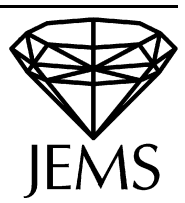

\title{
Stable ergodicity and julienne quasi-conformality
}

Received June 14, 1999 / final version received October 25, 1999

\begin{abstract}
In this paper we dramatically expand the domain of known stably ergodic, partially hyperbolic dynamical systems. For example, all partially hyperbolic affine diffeomorphisms of compact homogeneous spaces which have the accessibility property are stably ergodic. Our main tools are the new concepts - julienne density point and julienne quasi-conformality of the stable and unstable holonomy maps. Julienne quasi-conformal holonomy maps preserve all julienne density points.
\end{abstract}

\section{Introduction}

Let $M$ be a compact differentiable manifold without boundary and let $m$ be a smooth probability measure on $M$. We consider the space $\operatorname{Diff}_{m}^{2}(M)$ of $C^{2}$ diffeomorphisms of $M$ which are measure preserving (we also say volume preserving) with respect to the measure $m$. If there is a neighborhood $\mathscr{U}$ of $f$ in $\operatorname{Diff}_{m}^{2}(M)$ consisting entirely of $m$-ergodic diffeomorphisms, then $f$ is stably ergodic.

The persistence of positive measure sets of invariant tori due to Kolmogorov, Arnold, Moser, Herman and others (see Herman [13] and Yoccoz [27]) shows that stable ergodicity cannot in general be an open and dense property in $\operatorname{Diff}_{m}^{2}(M)$.

These invariant tori have no hyperbolic behavior at all. In contrast, a uniformly hyperbolic diffeomorphism $f \in \operatorname{Diff}_{m}^{2}(M)$ is stably ergodic. See Anosov's thesis [2]. In a series of papers, [12,20-23], we have been investigating the mixed situation which is partially hyperbolic.

Recall that $f: M \rightarrow M$ is partially hyperbolic if it is non-trivially normally hyperbolic in the following sense. The tangent bundle of $M$ is

Charles Pugh thanks IBM, Rockefeller University, and the Instituto de Matemática Pura e Aplicada for hospitality and support during part of the time this paper was written.

Michael Shub was supported in part by an NSF grant.

C. Pugh: Mathematics Department, University of California, Berkeley, CA, 94720, USA e-mail: pugh@math.berkeley.edu

M. Shub: IBM, Thomas J. Watson Research Center, Yorktown Heights, NY, 10598, USA e-mail:mshub@us.ibm.com

Mathematics Subject Classification (1991): 37C, 37D 
a $T f$-invariant direct sum

$$
T M=E^{u} \oplus E^{c} \oplus E^{s},
$$

the stable and unstable bundles $E^{s}$ and $E^{u}$ are non-zero, and $T f$ contracts and expands them more sharply than it does the center bundle $E^{c}$. That is, there is a Riemann structure on $T M$ such that $T^{u} f$ expands $E^{u}, T^{s} f$ contracts $E^{s}$, and

$$
\sup _{p}\left\|T_{p}^{s} f\right\|<\inf _{p} \mathbf{m}\left(T_{p}^{c} f\right) \text { and } \sup _{p}\left\|T_{p}^{c} f\right\|<\inf _{p} \mathbf{m}\left(T_{p}^{u} f\right),
$$

where $T^{u} f, T^{c} f, T^{s} f$ are the restrictions of $T f$ to $E^{u}, E^{c}, E^{s}$, and $\mathbf{m}(T)$ refers to the conorm of $T, \inf \{|T v|:|v|=1\}$. The center bundle is permitted to be zero, in which case $f$ is an Anosov diffeomorphism.

The center bolicity of $f$ is the ratio

$$
b=\frac{\left\|T^{c} f\right\|}{\mathbf{m}\left(T^{c} f\right)} .
$$

We say that $f$ is center bunched if $b$ is close to 1 . In a typical case, $b<1.09$ suffices. See Sect. 4 for details. The examples to which our stable ergodicity theory applies most readily are perturbations of cases where $b=1$, so we find center bunching a reasonable hypothesis.

Our main theme is: A little hyperbolicity goes a long way in guaranteeing stably ergodic behavior. In [21] we have stated

Conjecture 1. Stably ergodic diffeomorphisms are open and dense among the partially hyperbolic $C^{2}$ volume preserving diffeomorphisms of $M$.

An approach to this conjecture breaks it down into two parts, using the notion of accessibility.

Given $x, y \in M$ and given the splitting $T M=E^{u} \oplus E^{c} \oplus E^{s}$ for $f$, we say that $y$ is us-accessible from $x$ if there is a piecewise differentiable path joining $x$ to $y$ always tangent either to $E^{u}$ or $E^{s}$. Clearly, accessibility is an equivalence relation. If there is only one equivalence class (every $y$ is accessible from every $x$ ) we say that the splitting $E^{u} \oplus E^{s}$ (or the diffeomorphism $f$ ) has the accessibility property. If every $g$ in some neighborhood of $f$ has the accessibility property, $f$ is stably accessible. If the only measurable sets which are saturated by the accessibility equivalence relation have measure zero or one, we say that $E^{u} \oplus E^{s}$, or $f$, has the essential accessibility property. Obviously, stable accessibility implies accessibility implies essential accessibility.

Conjecture 2. Stable accessibility is an open and dense property among $C^{2}$ partially hyperbolic diffeomorphisms, volume preserving or not. Openness is obvious. 
Conjecture 3. Partially hyperbolic $C^{2}$ volume preserving diffeomorphisms with the essential accessibility property are ergodic.

As Anosov pointed out, much progress in dynamical systems theory relies on an improvement in our understanding of the properties of of the (un)stable manifold foliations. In this paper we introduce new concepts julienne density point and julienne quasi-conformality. We prove, using our center bunching conditions, that the holonomy maps of strong (un)stable foliations are julienne quasi-conformal and hence preserve all julienne density points. With this result we are able to dramatically weaken the additional hypotheses which we used in [21] to prove Conjecture 3. As a consequence, we markedly expand the class of known stably ergodic diffeomorphisms. For example, according to Theorem $\mathrm{C}$, below, all affine diffeomorphisms of compact homogeneous spaces with the accessibility property are stably ergodic.

Recall that $E^{u}$ and $E^{s}$ are tangent to unique foliations $\mathscr{W}^{u}$ and $\mathscr{W}^{s}$ which have $C^{1}$ leaves, but that since the bundles $E^{u}$ and $E^{s}$ are only Hölder continuous, the foliations may fail to be smooth. If $E^{u} \oplus E^{c}, E^{c}$, and $E^{c} \oplus E^{s}$ are also tangent to continuous foliations with $C^{1}$ leaves $\mathscr{W}^{c u}, \mathscr{W}^{c}$, and $\mathscr{W}^{c s}$ and if $\mathscr{W}^{c}$ and $\mathscr{W}^{u}$ subfoliate $\mathscr{W}^{c u}$, while $\mathscr{W}^{c}$ and $\mathscr{W}^{s}$ subfoliate $\mathscr{W}^{c s}$, then we say that $f$ is dynamically coherent.

Theorem A. If $f \in \operatorname{Diff}_{m}^{2}(M)$ is a center bunched, partially hyperbolic, dynamically coherent diffeomorphism with the essential accessibility property then $f$ is ergodic.

As a consequence we get

Corollary a. Center bunched partially hyperbolic diffeomorphisms which are stably dynamically coherent and stably accessible are stably ergodic.

Proof. According to [21], small perturbations preserve the properties of partial hyperbolicity and center bunching. Therefore, every perturbation of $f$ in $\operatorname{Diff}_{m}^{2}(M)$ satisfies the hypotheses of Theorem A and is ergodic.

We make two remarks. The first concerns the hypothesis in Corollary a that the diffeomorphism be stably dynamically coherent. Perhaps dynamical coherence is itself stable under perturbations of the diffeomorphism; we do not know. A general, stable condition that implies stability of dynamical coherence is plaque expansivity of the center foliation. For, as we showed in [14], a plaque expansive, normally hyperbolic foliation is structurally stable as a foliation, and this implies that dynamical coherence is unaffected by perturbations of the diffeomorphism. Also, if the center foliation happens to be $C^{1}$, or if the diffeomorphism acts isometrically on the center leaves, then plaque expansivity is automatic. 
Second, the hypothesis of partial hyperbolicity in Theorem A can be weakened somewhat. It is only necessary to assume that one of the two bundles $E^{u}, E^{s}$ is non-zero. This happens if the spectrum of $T f$ splits into just two parts, say an unstable part that represents the strong expanding behavior of $f$, and a remaining, indecomposable part that represents the weak expanding, neutral, and contracting behavior of $f$. Thus, $T f$ leaves invariant a splitting $T M=E^{u} \oplus E^{c}$. Since $M$ is compact, there must be some true contraction to balance the expansion in $E^{u}$. Some vectors in $E^{c}$ get contracted by $T f$, but there need not exist in this pseudo-center subbundle $E^{c}$ a continuous, invariant, purely contracting subbundle. The proof of Theorem A in this special case, however, is quite simple. Since $E^{s}=0$, a $u s$-path lies wholly in an unstable leaf. That is, accessibility equivalence classes are single unstable leaves. Now suppose that $f$ fails to be ergodic. Then there is an invariant set $A$ of intermediate measure, $0<m(A)<1$, and by the usual use of the Birkhoff Ergodic Theorem, it consists, except for a zero set, of almost whole unstable leaves. According to [19], the unstable foliation is absolutely continuous, even when $E^{s}=0$. Thus, when we form the unstable saturate of $A$, Sat ${ }^{u}(A)$, by taking the union of all unstable leaves that meet $A$ in sets of positive leaf measure, we see that $A$ differs from $\mathrm{Sat}^{u}(A)$ by a zero set. By construction $\mathrm{Sat}^{u}(A)$ is saturated by the accessibility equivalence relation, and this contradicts the hypothesis in Theorem A of essential accessibility.

The proof of Theorem A, proceeds by a careful examination of the measure theoretic regularity properties of the unstable holonomy map $h_{u}$, which is defined by sliding along the leaves of the unstable foliation. See Sect. 4. Also, of course, we have corresponding properties of the stable holonomy map $h_{s}$. Two facts are already known about $h_{u}$.

(a) $h_{u}$ is a bi-Hölder homeomorphism, but is not in general Lipschitz.

(b) $h_{u}$ has a continuous, positive Radon Nikodym derivative with respect to Lebesgue measure.

These facts were originally proved by Anosov in [2], (b) being his main technical tool to establish ergodicity. It implies that $h_{u}$ is absolutely continuous. See also [19]. To give it a name we say that a homeomorphism satisfying (b) is RN-regular. Automatically, the inverse of an $R N$-regular homeomorphism is $R N$-regular.

At issue is how the holonomy map $h_{u}$ affects density points. (Recall that $p$ is a Lebesgue density point of a set $S$ if the measure theoretic concentration of $S$ in the ball of radius $r$ at $p$ converges to 1 as $r \rightarrow 0$. See also Sect. 2.) In low dimensions or when the hyperbolic spectrum is tightly bunched, we showed in [12,21] that $h_{u}$ is density point preserving: $h_{u}$ carries every Lebesgue density point of a measurable set $S$ to a Lebesgue density point of $h(S)$. It is elementary that an $R N$-regular homeomorphism $h$ preserves 
almost all density points. In our proof of stable ergodicity, however, "almost all" is not enough. We need "all", and as we showed by example in [12], "all" is not implied by $R N$-regularity.

It is natural to ask whether $h_{u}$ satisfies some standard, additional property that implies it is density point preserving. For example, one easily checks that a bi-Lipschitz homeomorphism preserves density points. However, according to (a), above, holonomy maps are not in general bi-Lipschitz. A different criterion is quasi-conformality. In [11] Gehring and Kelley prove that a bi-quasi-conformal homeomorphism is density point preserving. It turns out, though, that

$$
\text { bi-RN + bi-quasi-conformal } \Rightarrow \text { bi-Lipschitz, }
$$

so we can not hope that the Gehring-Kelley Theorem applies to holonomy maps in general. (This implication is a nice exercise in pictorial measure theory.)

At present, we are unable to say whether holonomy preserves Lebesgue density points in general. Rather, using shapes called juliennes in place of round balls (see Sect. 4), we re-define the density point concept and prove three things.

(a) Almost every Lebesgue density point is a julienne density point, and vice versa.

(b) The holonomy maps are quasi-conformal with respect to juliennes.

(c) The holonomy maps preserve (all) julienne density points.

In fact, (b) implies (c), which is a generalization of the Gehring-Kelley Theorem. See Sect. 8. From all this, we draw the conclusion that

\section{Juliennes are the natural shapes to use when analyzing holonomy maps.}

We began studying juliennes in [12] and continued to investigate their properties in [21]. The difference in our current approach is that we control the shape of the juliennes dynamically rather than only through Hölder estimates on the $E^{u}$ and $E^{s}$ bundles. This is carried out in Sects. 4 and 6.

In some ways it seems a shame to give up the infinitesimal information embodied in $E^{u}$ and $E^{s}$ and to rely on more ad hoc geometric constructions. Anosov (pages 127-129 and 167 of [2]) voices a similar sentiment regarding the existence and uniqueness of stable and unstable manifolds: instead of ad hoc dynamical arguments, he imagines there should be a construction that uses non-differentiable Frobenius integrability conditions, and avoids iteration.

It can be shown that the holonomy maps have a kind of flag differentiability. Perhaps julienne quasi-conformality can be derived directly from this infinitesimal property. 
Once we have proven the density point preservation properties for stable and unstable holonomy maps, the proof of Theorem A proceeds as in [12,21] along classical lines dating from Hopf [15], Anosov [2], and others, using $u s$-accessibility and the essential $\mathscr{W}^{u}$ and $\mathscr{W}^{s}$ saturation of invariant sets. There are, however, some subtleties to this proof, one of which we discuss now.

The center foliation, when it exists, integrates the center plane field $E^{c}$. It is even less regular than the stable and unstable foliations, which always exist. As has been shown by A. Katok in an example written up by J. Milnor [16], the center foliation for a smooth system can have the following measure theoretically singular property: for some measurable set $S$ of full measure, each center leaf meets $S$ in at most one point. This phenomenon has been referred to as "Fubini's nightmare", or, by Milnor, as "Fubini Foiled". Even though the center leaves are smooth, and the center plane field is Hölder continuous, the center foliation is unavoidably pathological.

Theorem A allows us to deduce $C^{2}$ stable ergodicity for many affine diffeomorphisms of compact homogeneous spaces, as we now explain. See also Sect. 10. We make the following

\section{Standing Lie Group Hypothesis.}

(a) $G$ is a connected Lie group and $B \subset G$ is a proper, closed subgroup.

(b) $G / B$ is compact.

(c) Haar measure projects to a finite measure $m$ on $G / B$, invariant under left translation, $x B \mapsto g x B$.

(d) $f: G / B \rightarrow G / B$ is an affine diffeomorphism. That is, it is part of a commutative diagram

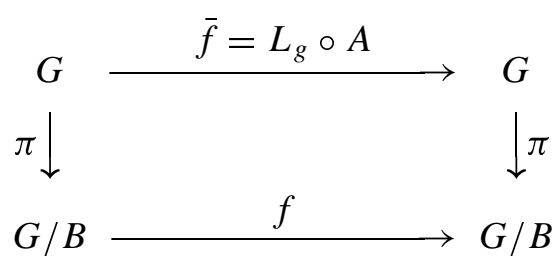

where $A$ is an automorphism of $G, L_{g}$ is left multiplication by some fixed element $g \in G$, and, to make the projection well defined, $A(B)=B$.

A simple example is $G=\mathbb{R}^{m}$ and $B=\mathbb{Z}^{m}$. Then $G / B$ is the torus $\mathbb{T}^{m}$. The map $L_{g}$ is an affine translation of $\mathbb{R}^{m}$ to itself, $A$ is an $m \times m$ integer matrix with determinant \pm 1 , and $f$ sends the coset $[x]$ to the coset $[g+A x]$.

The hypotheses of Theorem A for a general affine diffeomorphism $f$ are easily checked at the Lie algebra level. The Lie algebra of $G$ is $\mathfrak{g}=T_{e} G$, 
where $e$ is the identity element of $G$. The adjoint action of $g$ on $\mathfrak{g}$ is given by the derivative at the identity of $g$-conjugation,

$$
\begin{aligned}
\operatorname{Ad}(g): & \mathfrak{g} \rightarrow \mathfrak{g} \\
X & \mapsto T_{g} R_{g^{-1}} \circ T_{e} L_{g}(X),
\end{aligned}
$$

where $R_{g^{-1}}$ is right multiplication by $g^{-1}, R_{g^{-1}}: h \mapsto h g^{-1}$. Induced by $\bar{f}$ is a Lie algebra automorphism

$$
\mathfrak{a}(\bar{f})=\operatorname{Ad}(g) \circ T_{e} A .
$$

It determines when we can apply Theorem A to $f$ as follows. Split $\mathfrak{g}=T_{e} G$ according to the generalized eigenspaces of $\mathfrak{a}(\bar{f})$,

$$
\mathfrak{g}=\mathfrak{g}^{u} \oplus \mathfrak{g}^{c} \oplus \mathfrak{g}^{s},
$$

where the eigenvalues of $\mathfrak{a}(\bar{f})$ on $\mathfrak{g}^{u}, \mathfrak{g}^{c}, \mathfrak{g}^{s}$ have modulus $>1,=1$, and $<1$ respectively. As we show in Sect. 10, these eigenspaces are Lie subalgebras. See also [21]. The hyperbolic subalgebra of $\bar{f}$ is the smallest Lie subalgebra $\mathfrak{h}$ such that $\mathfrak{g}^{u} \cup \mathfrak{g}^{\mathfrak{s}} \subset \mathfrak{h} \subset \mathfrak{g}$. It is an ideal in $\mathfrak{g}$. See [21]. Let $\mathfrak{b}$ be the Lie algebra of $B, \mathfrak{b} \subset \mathfrak{g}$.

Theorem B. Suppose that $f$ is an affine diffeomorphism of the compact homogeneous space $G / B$. Then

(a) $f$ is partially hyperbolic if and only if $\mathfrak{h} \not \subset \mathfrak{b}$.

(b) If $f$ is partially hyperbolic then it is center bunched and dynamically coherent.

(c) $f$ has the accessibility property if and only if $\mathfrak{g}=\mathfrak{b}+\mathfrak{h}$.

We deduce

Theorem C. The affine diffeomorphism $f: G / B \rightarrow G / B$ is stably ergodic among $C^{2} m$-preserving diffeomorphisms of $G / B$ if (merely) the hyperbolic Lie subalgebra $\mathfrak{h}$ is large enough that $\mathfrak{g}=\mathfrak{b}+\mathfrak{h}$.

Here are some special cases of Theorem C. For their proofs and that of Theorem C, see Sect. 10.

Corollary b. Suppose that $G$ is a simple Lie group (its Lie algebra has no non-trivial ideals), $B$ is a uniform discrete subgroup of $G$, the homogeneous space $G / B$ is compact, and $g \in G$ is given. Left translation by $g$ on $G / B$ is stably ergodic among $C^{2}$ volume preserving diffeomorphisms if and only if $A d(g)$ has at least one eigenvalue of modulus different from 1. 
Corollary c. Let $B$ be a uniform discrete subgroup of $S L(n, \mathbb{R})$ and let $M \in S L(n, \mathbb{R})$ be given. Left multiplication by $M$ is stably ergodic among $C^{2}$ volume preserving diffeomorphisms of $S L(n, \mathbb{R}) / B$ if and only if $M$ has at least one eigenvalue of modulus different than 1.

These corollaries provide examples of what we mean by a little hyperbolicity going a long way toward guaranteeing stable ergodicity. The hyperbolic part of $M$ can be low dimensional, the center part high dimensional, and nevertheless we get stable ergodicity.

See [21] for more equivalent conditions to stable ergodicity for $S L(n, \mathbb{R})$ and semi-simple Lie groups.

The first case of Corollary c not covered by [21] is given by $S L(3, \mathbb{R})$ and a matrix $M$ with two eigenvalues of modulus different than one and the third equal to one. In this case the hyperbolic bunching conditions of [21] are violated.

To our knowledge ergodicity in the context of partial hyperbolicity and accessibility was first considered by Brin and Pesin [6]. Brin in [5] goes further in this direction. It seems quite likely that the open and dense set of skew products he considers in [5] which are stably ergodic among skew products are also stably ergodic in Diff ${ }_{m}^{2}$, but we have not verified it. The preprint of Burns and Wilkinson [8] is relevant here. Further work on skew products is contained in papers by Adler, Kitchens, and Shub [1], Field and Parry [10], and Parry and Pollicott [18]. For stable ergodicity of time one maps of geodesic flows see the the papers of Grayson Pugh and Shub [12], Wilkinson [26], and Burns, Pugh, and Wilkinson [7].

We thank Dennis Sullivan for several helpful conversations about density points. It was in his seminar at CUNY that we first became accustomed to such concepts as bounded distortion and quasi-conformality in relation to density points.

\section{Density points}

The technical basis for our main theorems is differentiation of integrals. See M. de Guzmán's book, Differentiation of integrals in $\mathbb{R}^{n}$, [9] and Chapt. 1 of E. Stein's book, Harmonic Analysis [25]. In this section we review some of the ideas involved.

Let $X$ be a locally compact metric space and let $m$ be a regular, nonatomic, locally finite, Borel measure on it. The measure of a measurable set $S \subset X$ is the infimum of the measures of open sets that contain $S$. Points have zero measure. Compact sets have finite measure. For example, $m$ could be a smooth measure on a manifold. A family $\mathscr{T}=\bigcup \mathscr{T}_{p}$, indexed by $p \in X$, is a Vitali basis if each $\mathscr{T}_{p}$ is non-empty, the sets 
$V \in \mathscr{T}_{p}$ are measurable with non-zero measure (hence non-zero diameter), each contains $p$, and there exist in $\mathscr{T}_{p}$ sets of arbitrarily small diameter. The simplest good example is the family $\mathscr{B}$ of all $n$-dimensional balls, of all radii, centered at all points of $\mathbb{R}^{n}$.

The concentration, or conditional measure, of a measurable set $A$ in a measurable set $V$ of non-zero measure, is the ratio

$$
[A: V]=\frac{m(A \cap V)}{m(V)} .
$$

Fix a Vitali basis $\mathscr{V}$. A point $p \in X$ is a $\mathscr{V}$-density point of $A$ if

$$
\lim [A: V]=1,
$$

the limit being taken as $V \in \mathscr{T}_{p}$ shrinks to $p$. The basic fact about density points is the

Lebesgue-Vitali Theorem. With respect to the Vitali basis $\mathscr{B}$ of $n$-balls, almost every point $p$ of a measurable set $A \subset \mathbb{R}^{n}$ is a density point of $A$.

In this paper we need to generalize the Lebesgue-Vitali theorem to Vitali bases more dynamically natural than $\mathscr{B}$. We must work not with balls, but with juliennes, small sets that are long and thin. They look like slivered vegetables. At smaller and smaller scales, our juliennes become less round, thinner, and more elongated. See Sect. 4 in this paper. One of our main results is that despite the juliennes' strange shape, almost every point of a Lebesgue measurable set is a julienne density point. See Theorem 8.4.

The Vitali basis $\mathscr{V}$ differentiates the integral of an integrable function $\phi: X \rightarrow \mathbb{R}$ if, for almost all points $p \in X$,

$$
\phi(p)=\lim \frac{1}{m(V)} \int_{V} \phi(x) d x .
$$

The limit is taken as the set $V \in \mathscr{T}_{p}$ shrinks to $p$; i.e., diam $V \rightarrow 0$. If $\mathscr{T}$ differentiates the integral of every $\phi$ in some set $\Phi$ of locally integrable functions, we say that $\mathscr{T}$ is a $\Phi$-differentiation basis.

The Lebesgue-Vitali Theorem can be restated as the fact that $\mathscr{B}$ is a $\chi$-differentiation basis, where $\chi$ is the set of characteristic functions of measurable subsets of $\mathbb{R}^{n}$. For

$$
[A: V]=\frac{1}{m(V)} \int_{V} \chi_{A}(x) d x .
$$

In fact more is true. As Lebesgue showed, $\mathscr{B}$ is an $\mathscr{D}_{\text {loc }}^{1}$-differentiation basis.

If $\mathscr{V}$ is a $\chi$-differentiation basis, it is called a density basis. A result of Busemann and Feller shows that being a density basis is equivalent to being an $\mathscr{L}_{\text {loc }}^{\infty}$-differentiation basis. See [9], page 72. 
The geometric characterization of density bases in $\mathbb{R}^{n}$ is a central point in much of analysis (see [25]), and has a long history (see [9]).

When balls are replaced by cubes (whose edges need not be parallel to the coordinate axes), the same result holds: The cubic Vitali basis is a density basis. But even when cubes are replaced by $n$-dimensional rectangles the situation becomes subtle and is not completely understood. There is a tradeoff between the eccentricity of the rectangles and how much their edge directions vary.

First consider the case in which the edge directions do not vary at all. The Vitali basis $\mathscr{R}_{a}$ of $n$-dimensional rectangles whose edges are axis parallel is a density basis, but it is not an $\mathscr{L}_{\text {loc }}^{1}$-differentiation basis. See [9], pages 74 and 96 . To refine $\mathscr{R}_{a}$ so that it becomes an $\mathscr{D}_{\text {loc }}^{1}$-differentiation basis we impose a restriction on the eccentricity of the rectangles. A sufficient condition is that for some constant $K>0$ and all rectangles $R$ of diameter $\leq 1$,

$$
\text { eccentricity } R \leq(\text { diameter } R)^{-K} \text {. }
$$

For this type of condition implies the "doubling property" of Stein [25], page 8 , or the "volumetric engulfing property" discussed in Sect. 3 below.

It is easy to see that the particular choice of coordinate axes is irrelevant. If $\mathscr{R}_{a^{\prime}}$ is the Vitali basis of rectangles whose edges are always parallel to some fixed, finite set of directions in $\mathbb{R}^{n}$ then $\mathscr{R}_{a^{\prime}}$ is a density basis, and if the eccentricities of its rectangles are bounded by a fixed, negative power of their diameter then $\mathscr{R}_{a^{\prime}}$ becomes an $\mathscr{D}_{\text {loc }}^{1}$-differentiation basis.

On the other hand, when the edge directions of rectangles in a Vitali basis are drawn from an infinite set, the situation is subtler. As is shown by Nikodym's "paradoxical set" [17], the Vitali basis $\mathscr{R}$ of all $n$-dimensional rectangles is not a density basis. Nor does $\mathscr{R}$ become a density basis if one imposes a continuity restriction on the edge directions. See Chapt. 5 of de Guzmán's book [9].

The Vitali bases discussed so far are linear. Rectangles are linear images of the cube. As we stated before, however, the Vitali bases that arise naturally in non-linear dynamics consist of juliennes - non-linear rectangloids with varying edge directions. Despite the obstacles described above, juliennes turn out to be satisfactory Vitali bases due, it seems, to three factors.

(a) The elongation axes of a julienne are Hölder controlled, not merely continuously controlled.

(b) The eccentricity of a julienne and its diameter are both exponential functions of a common number $n$, the number of dynamical iterations.

(c) The non-linearity, nesting, and shape-scaling properties of the julienne are governed by a fixed, smooth dynamical system.

One might hope to get an abstract geometric characterization of density bases, say for a basis $\mathscr{V}$ of rectangles in $\mathbb{R}^{2}$, in these sorts of terms. If the 
eccentricity of rectangles in $\mathscr{V}$ is no greater than a fixed negative power $-K$ of the diameter, if the edge directions of rectangles in $\mathscr{T}_{x}$ are a $\theta$-Hölder function of $x$, and if there is some favorable relation between $K$ and $\theta$ (say $K(1-\theta)<1)$, does it follow that $\mathscr{T}$ is a density basis?

We conclude this section by discussing a transformation property of density points.

Let $(X, m)$ and $\left(X^{\prime}, m^{\prime}\right)$ be measure spaces of the type we have been considering (locally compact, etc.) and let $\mathscr{T}, \mathscr{T}^{\prime}$ be Vitali bases on them. Let $h: X \rightarrow X^{\prime}$ be a homeomorphism. If $h$ bijects $\mathscr{T}$ to $\mathscr{V}^{\prime}$, and has a positive, continuous Radon Nikodym derivative it is easy to see that $\mathscr{V}$ density points biject to $\mathscr{T}^{\prime}$-density points. We need a slightly better version of this result. We say that the homeomorphism $h$ is K-quasi-conformal with respect to $\mathscr{T}, \mathscr{T}^{\prime}$ if for all small $V \in \mathscr{T}_{p}$, there are small $V^{\prime}, V^{\prime \prime} \in \mathscr{T}_{h(p)}^{\prime}$ such that

$$
V^{\prime} \subset h(V) \subset V^{\prime \prime} \quad \text { and } \quad \frac{m^{\prime}\left(V^{\prime \prime}\right)}{m^{\prime}\left(V^{\prime}\right)} \leq K .
$$

Ordinary quasi-conformality in $\mathbb{R}^{n}$ is the same as quasi-conformality with respect to the Vitali basis of round balls and Lebesgue measure.

Proposition 2.1. Assume that $h$ and $h^{-1}$ are quasi-conformal with respect to the Vitali bases $\mathscr{V}, \mathscr{V}^{\prime}$. If $h$ has a continuous, positive Radon Nikodym derivative then it bijects $\mathscr{V}$-density points to $\mathscr{V}^{\prime}$-density points.

Proof. Suppose that $p \in X$ is not a density point of $A \subset X$. Then, for some $\epsilon>0$ and some $V \in \mathscr{T}_{p}$ with arbitrarily small diameter, $[A: V] \leq 1-\epsilon$. By continuity of the Radon Nikodym derivative and smallness of $V,[h(A)$ : $h(V)] \leq 1-\epsilon / 2$. Since $h(V)$ occupies at least a portion $1 / K$ of $V^{\prime \prime}$, the concentration of $h(A)$ in $V^{\prime \prime}$ is bounded away from 1. That is, $h(p)$ is not a density point of $h(A)$.

We have shown that $h$ carries non-density points to non-density points. The same is true for $h^{-1}$. Hence $h$ and $h^{-1}$ also biject density points.

\section{Volumetric engulfing}

In this section we prove a version of the Lebesgue-Vitali density point theorem. We will apply the result to juliennes in Sect. 8.

As in the previous section, let $X$ be a locally compact, metric measure space, and let $m$ be a regular, non-atomic, locally finite, Borel measure on it. Let $\mathscr{T}$ be a Vitali basis on $X$. We also assume that $\mathscr{V}$ is filtered by a rank function

$$
\text { rank }: \mathscr{T} \rightarrow \mathbb{N}
$$


compatible with diameter in the sense that $\operatorname{rank}(V) \rightarrow \infty$ if and only if $\operatorname{diam}(V) \rightarrow 0$. That is, given $\epsilon>0$, there is an integer $N$ such that if $\operatorname{rank}(V) \geq N$ then $\operatorname{diam}(V)<\epsilon$, and conversely, given $N \in \mathbb{N}$ there is an $\epsilon>0$ such that if $\operatorname{diam}(V)<\epsilon$ then $\operatorname{rank}(V) \geq N$.

Definition. $\mathscr{V}$ is volumetrically engulfing if for some constant $L$, and for each $V \in \mathscr{V}$ there is a measurable set $\widehat{V}$ containing $V$ such that

(a) $m(\widehat{V}) \leq L m(V)$.

(b) If $V, V^{\prime} \in \mathscr{T}$ have non-empty intersection and $\operatorname{rank}(V) \leq \operatorname{rank}\left(V^{\prime}\right)$ then $V^{\prime} \subset \widehat{V}$.

(c) If $m(V) \rightarrow 0$ then $\operatorname{rank}(V) \rightarrow \infty$. That is, given $N \in \mathbb{N}$ there exists $\delta>0$ such that $m(V)<\delta$ implies $\operatorname{rank}(V) \geq N$.

See Fig. 1

$\mathscr{T}$ is closed if every $V \in \mathscr{T}_{x} \in \mathscr{T}$ is a closed subset of $X$.

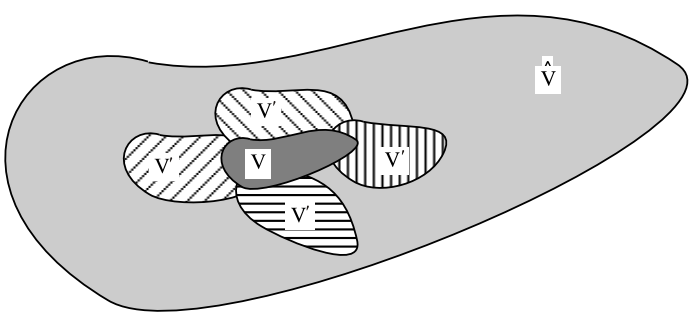

Fig. 1. The set $\widehat{V}$ engulfs all $V^{\prime} \in \mathscr{V}$ that meet $V$ but are not much larger than $V$

Theorem 3.1. If $\mathscr{V}$ is a volumetrically engulfing closed Vitali basis of $X$ and $S$ is a measurable subset of $X$ then almost every point of $S$ is a density point of $S$ with respect to $\mathscr{T}$. In other words, $\mathscr{V}$ is a density basis.

Proof. For each $\beta<1$ consider the set

$$
B=B_{\beta}=\{x \in S: \liminf [S: V]<\beta\} .
$$

The liminf is taken as $V \in \mathscr{T}_{p}$ shrinks to $p$. We claim that $B$ has measure zero. From this the theorem follows, for we can take a sequence $\beta_{n}=1-1 / n$ that tends to 1 . Almost every point of $S$ belongs to $S^{\prime}=S \backslash \bigcup B_{\beta_{n}}$, and every $x \in S^{\prime}$ is a density point of $S$.

Let $\epsilon>0$ be given. It is enough to show that $B$ has measure $<\epsilon$. Cover $B$ by an open set $U$ such that

$$
m(U)<m(B)+\frac{\epsilon(1-\beta)}{\beta} .
$$


Define a Vitali covering of $B$ by

$$
\mathscr{W}=\{V \in \mathscr{T}: V \subset U, \operatorname{diam}(V) \leq 1, \text { and }[S: V]<\beta\} .
$$

Since $B \subset S$,

$$
[B: V] \leq[S: V] .
$$

Apply the standard method of proof of the Lebesgue-Vitali Theorem. First choose a $V_{1} \in \mathscr{W}$ with minimum rank. All $V \in \mathscr{W}$ have $\operatorname{rank}(V) \geq$ $\operatorname{rank}\left(V_{1}\right)$. Then choose a $V_{2} \in \mathscr{W}$ with minimum rank among those members of $\mathscr{W}$ that do not meet $V_{1}$. Continue inductively. This produces a sequence of disjoint sets $V_{n}$ contained in $U$. Their total measure is no more than the measure of $U$. In each $V_{n}$, the concentration of $B$ is $<\beta$. There may be some points of $B$ which fail to be covered by the sets $V_{n}$. Call this not-covered set

$$
B^{\prime}=B \backslash \bigcup V_{n}
$$

We claim that $B^{\prime}$ has outer measure zero. (We are still in the process of showing that $B$ has measure $<\epsilon$.) Since the series $\sum m\left(V_{n}\right)$ converges, its tail, $\sum_{n>N} m\left(V_{n}\right)$, tends to zero as $N \rightarrow \infty$. To prove that $B^{\prime}$ has outer measure zero, then, it is enough to show that for each $N \in \mathbb{N}, B^{\prime}$ is contained in $\bigcup_{n>N} \widehat{V}_{n}$. For since $\mathscr{T}$ is volumetrically engulfing, the series $\sum_{n>N} m\left(\widehat{V}_{n}\right)$ is no greater than $L \sum_{n>N} m\left(V_{n}\right)$, which tends to 0 as $N \rightarrow \infty$.

Since $\sum m\left(V_{n}\right)$ converges, its terms tend to zero. According to (c) in the definition of volumetric engulfing, $\operatorname{rank}\left(V_{n}\right) \rightarrow \infty$.

Fix an $N$ and consider a point $x \in B^{\prime}$. Since $x \notin \bigcup_{n=1}^{N} V_{n}$, since the sets $V_{n}$ are closed, and since the sets in $\mathscr{T}_{x}$ have arbitrarily small diameter, there is a set $V \in \mathscr{W}$ which contains $x$ and is disjoint from $V_{1}, \ldots, V_{N}$. The set $V$ was available for choice, but was never chosen. It has finite rank. Therefore the reason it was never chosen is that for some first $V_{i}, i>N$, $V_{i} \cap V \neq \varnothing$. Since we always choose a set with minimum rank, and since $V$ was available to be chosen, we see that $\operatorname{rank}\left(V_{i}\right) \leq \operatorname{rank}(V)$. Because $\mathscr{V}$ is volumetrically engulfing, the set $\widehat{V}_{i}$ engulfs $V$. That is,

$$
B^{\prime} \subset \bigcup_{n>N} \widehat{V}_{n},
$$

and it follows that $B^{\prime}$ has outer measure zero.

Except for the zero set $B^{\prime}, B$ is covered by the disjoint sets $V_{n}$, and in each its concentration is at most $\beta$. Thus

$$
\begin{aligned}
m(B) & =\sum m\left(V_{n} \cap B\right) \leq \beta \sum m\left(V_{n}\right) \\
& \leq \beta m(U)<\beta\left(m(B)+\frac{\epsilon(1-\beta)}{\beta}\right) \\
& =\beta m(B)+\epsilon(1-\beta) .
\end{aligned}
$$


Therefore, $(1-\beta) m(B)<\epsilon(1-\beta)$ and $m(B)<\epsilon$. Since $\epsilon$ is arbitrary, it follows that $B$ has measure zero, and as observed at the outset, this implies that almost every point of $S$ is a density point with respect to $\mathscr{V}$.

Corollary 3.2. If $\mathscr{V}$ is a volumetrically engulfing closed Vitali basis of $X$ and $S \subset X$ is measurable, then for almost every point $x \in X$, the concentration of $S$ in $V$ converges to the characteristic function of $S$ at $x$ as $V \in \mathscr{V}_{x}$ shrinks to $x$,

$$
\lim [S: V]= \begin{cases}1 & \text { if } x \in S \\ 0 & \text { if } x \notin S .\end{cases}
$$

Proof. Theorem 3.1 applies equally to $S$ and to its complement $S^{c}=X \backslash S$. Thus, for almost every point $x \in X$,

$$
\begin{aligned}
& \text { if } x \in S \text { then }[S: V] \rightarrow 1 \text { as } V \in \mathscr{T}_{x} \text { shrinks to } x . \\
& \text { if } x \in S^{c} \text { then }\left[S^{c}: V\right] \rightarrow 1 \text { as } V \in \mathscr{V}_{x} \text { shrinks to } x .
\end{aligned}
$$

Measurability implies that $[S: V]+\left[S^{c}: V\right]=1$, and the corollary follows.

It is easy to phrase the volumetrically engulfing concept in terms of diameter, not rank. Namely, for some $L, K$,

(a) $m(\widehat{V}) \leq L m(V)$.

(b') If $V, V^{\prime} \in \mathscr{T}$ have non-empty intersection and $\operatorname{diam}\left(V^{\prime}\right) \leq K \operatorname{diam}(V)$ then $V^{\prime} \subset \widehat{V}$.

(c') If $m(V) \rightarrow 0$ then $\operatorname{diam}(V) \rightarrow 0$.

Thus, our volumetric engulfing condition is the same as assumption (i,ii)* of [25], page 8 . This makes Theorem 3.1 a consequence of the corollary of [25], page 13. The proof given there involves maximal functions.

\section{Juliennes}

Juliennes are foliation product sets constructed as follows. Let $\mathscr{W}^{u}, \mathscr{W}^{c u}$, $\mathscr{W}^{c}, \mathscr{W}^{c s}, \mathscr{W}^{s}$ be dynamically coherent foliations. At $p \in M$ we will choose

$$
X \subset W_{\mathrm{loc}}^{u}(p) \quad D \subset W_{\mathrm{loc}}^{c}(p) \quad Y \subset W_{\mathrm{loc}}^{s}(p)
$$

with carefully estimated small sizes. Then the center unstable julienne, the center stable julienne, and the solid julienne are

$$
\begin{aligned}
& J^{c u}=W^{c \cap u}(X \cup D) \\
& J^{c s}=W^{c \cap s}(D \cup Y) \\
& J^{\text {solid }}=W^{u \cap s}\left(J^{c u} \cup J^{c s}\right),
\end{aligned}
$$


where the notation $W^{a \cap b}(C)$ refers to the set of all intersection points of local $a$-manifolds and local $b$-manifolds that pass through $C$. That is,

$$
W^{a \cap b}(C)=\left\{z: \text { for some } c, c^{\prime} \in C, z \in W_{\mathrm{loc}}^{a}(c) \cap W_{\mathrm{loc}}^{b}\left(c^{\prime}\right)\right\} .
$$

Equivalently,

$$
W^{a \cap b}(C)=W_{\mathrm{loc}}^{a}(C) \cap W_{\mathrm{loc}}^{b}(C) .
$$

See Figs. 2 and 3. We refer to $W^{a \cap b}(C)$ as the (local) $\mathbf{a} \cap \mathbf{b}$-saturate of $C$. The permitted vaues of $a, b$ are $u, c, s$, and we assume that $a \neq b$. Also, we assume that the local leaves have small enough radius $r$ so that if $\operatorname{diam} C \leq r$ and $c, c^{\prime} \in C$ then

$$
W^{a}(c, r) \cap W^{b}\left(c^{\prime}, r\right)=W^{a}(c, 2 r) \cap W^{b}\left(c^{\prime}, 2 r\right) .
$$

(Since $\mathscr{W}^{a}, \mathscr{W}^{b}$ are non-tangential, these intersections are both empty or both a single point.) From this it follows that local $a b$-saturation is idempotent: if $C$ has small diameter then

$$
W^{a \cap b}\left(W^{a \cap b}(C)\right)=W^{a \cap b}(C) .
$$

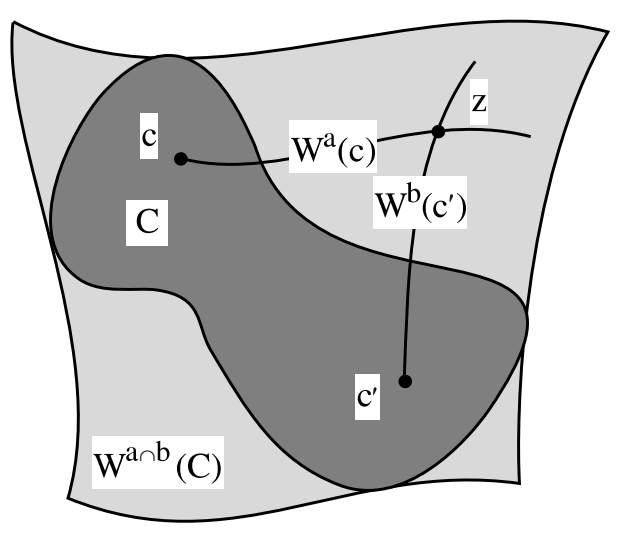

Fig. 2. $W^{a \cap b}(C)$ fills out $C$ by the local foliations $\mathscr{W}^{a}, \mathscr{W}^{b}$

We call $J \cap W_{\text {loc }}^{a}(z)$ the a-fiber of the julienne through the point $z \in J$. In particular, if $z \in D$, the unstable fiber of $J^{c u}$ through $z$ is denoted as $X(z)$, while the stable fiber of $J^{c s}$ is $Y(z)$. If $z=p$ then $X(z)=X$ is the main unstable fiber of $J^{c u}$ while $Y(z)=Y$ is the main stable fiber of $J^{c s}$. We call the center fiber $D$ the center core of the julienne. 


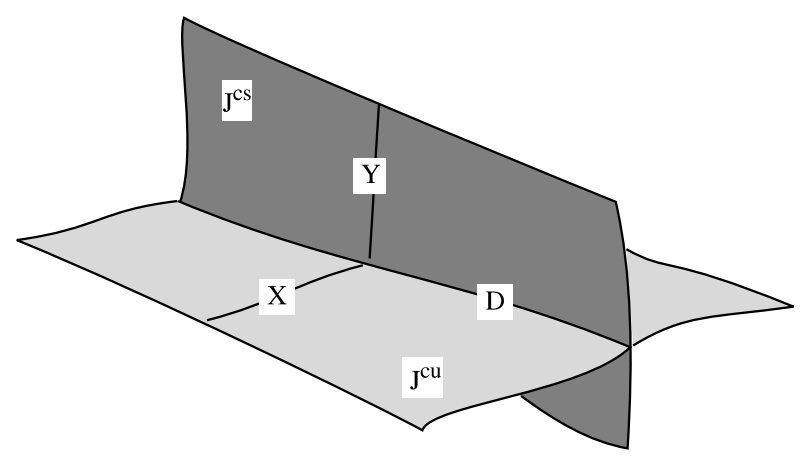

Fig. 3. The solid julienne is the $u \cap s$-saturate of $J^{c u} \cup J^{c s}$

Proposition 4.1. Juliennes are natural in three ways. They are locally maximal, they iterate naturally under $f$, and they behave correctly under holonomy.

Proof. Juliennes are of the form

$$
J=W^{a \cap b}(C)
$$

for $a b=c u$ and $C=X \cup D, a b=c s$ and $C=D \cup Y$, or $a b=u s$ and $C=J^{c u} \cup J^{c s}$. By local maximality we mean that

$$
J=W^{a \cap b}(J),
$$

which is the statement that local $a b$-saturation is idempotent.

By naturality under $f$ iteration we mean that for $J=W^{a \cap b}(C)$, we have

$$
f(J)=W^{a \cap b}(f(C)),
$$

a fact that is clear from invariance of the $a$ - and $b$-foliations.

Naturality under holonomy applies primarily to the center unstable and center stable juliennes. We assert that these juliennes are products in the following sense.

(a) The center holonomy gives a homeomorphism $Y(z) \rightarrow Y\left(z^{\prime}\right)$ between any two stable fibers of $J^{c s}$. It also gives a homeomorphism $X(z) \rightarrow$ $X\left(z^{\prime}\right)$ between any two unstable fibers of $J^{c u}$.

(b) The unstable holonomy gives a homeomorphism between any two center fibers of $J^{c u}$, while the stable holonomy gives a homeomorphism between any two center fibers of $J^{c s}$.

These holonomy facts are a direct consequence of our dynamical coherence assumption: the unstable and center foliations sub-foliate the center unstable foliation, while the center and stable foliations sub-foliate the center stable foliation. 
Our choice $X, D, Y$ involves several estimates on Hölder exponents, contraction rates, etc. We start by defining

$$
\begin{aligned}
\mu & =\min \left\{\mathbf{m}(T f), \mathbf{m}\left(T f^{-1}\right)\right\} \\
\nu & =\max \left\{\left\|T^{s} f\right\|,\left\|T^{u} f^{-1}\right\|\right\} \\
\gamma & =\min \left\{\mathbf{m}\left(T^{c} f\right), \mathbf{m}\left(T^{c} f^{-1}\right)\right\} .
\end{aligned}
$$

The assumption of partial hyperbolicity implies

$$
0<\mu \leq \nu<\gamma \leq 1
$$

The behavior of $f$ in the stable direction is a contraction whose strength is between $\mu$ and $\nu$, the behavior of $f$ in the unstable direction is an expansion whose strength is between $1 / \nu$ and $1 / \mu$, and the behavior of $f$ in the center direction is an isometry up to a factor between $\gamma$ and $1 / \gamma$.

The Fiber Contraction Theorem of [14], pages 30 and 81, and [24], page 45, converts this expansion/contraction information into regularity statements. For example, to estimate the Hölder exponent of $E^{c u}$ we proceed as follows. Let $L$ be the vector bundle over $M$ whose fiber at $p \in M$ is the space of linear maps $S: E_{p}^{c u} \rightarrow E_{p}^{s}$,

$$
L_{p}=L\left(E_{p}^{c u}, E_{p}^{s}\right) .
$$

The graph transform sends $S \in L_{p}$ to $(T f)_{\#} S \in L_{f(p)}$ where

$$
\operatorname{graph}\left((T f)_{\#} S\right)=(T f)(\operatorname{graph}(S)) .
$$

This gives a bundle map

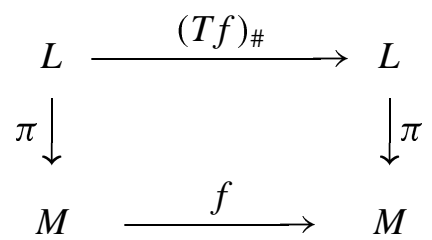

in which the fiber is contracted more sharply than $v \gamma^{-1}$, while the base contraction is at worst $\mu$. (We use the operator norm on fibers and the Riemann metric on the base.) The zero section of $L$ is its unique invariant section, and it is $\theta$-Hölder if the fiber contraction $\theta$-dominates the base contraction in the sense that

$$
\nu \gamma^{-1} \mu^{-\theta}<1 .
$$

(In the actual proof of this, to avoid circular reasoning we smoothly approximate $E^{c u}, E^{s}$ by bundles $\widetilde{E}^{c u}, \widetilde{E}^{s}$, we replace $L$ by $\widetilde{L}=L\left(\widetilde{E}^{c u}, \widetilde{E}^{s}\right)$, and we restrict the graph transform to the unit disc bundle of $\widetilde{L}$.) 
Since our expansion/contraction hypotheses are symmetric, the same estimate is valid for the Hölder exponent of $E^{c s}$, and hence for the center bundle $E^{c}=E^{c u} \cap E^{c s}$. In fact the estimate holds also for the unstable and stable bundles, and, manipulating (6), we see that all five bundles $E^{u}, \ldots, E^{s}$ are $\theta$-Hölder if

$$
\theta<\frac{\log \left(v \gamma^{-1}\right)}{\log \mu} .
$$

From now on, $\theta$ will denote the Hölder exponent of $E^{c}, 0<\theta \leq 1$. It is no smaller than the $\theta$ estimated in (7), but by good fortune (for instance, if $f$ is affine) it might be larger. In terms of this $\theta$ we will assume the following center bunching condition

$$
v<\gamma^{2+2 / \theta} .
$$

Thus, $\gamma$ is so near 1 that even a potentially high power of it remains greater than $v$. Center bunching means that hyperbolic behavior strongly dominates center behavior.

Perturbations of the affine diffeomorphisms discussed in Sect. 1 satisfy the center bunching hypothesis. See Sect. 10.

It may be of interest to express the center bunching condition in a way that depends only on $\mu, v$.

Proposition. If $\mu=v^{r}, r \geq 1$, then sufficient for the center bunching condition (8) is that $\gamma$ is so close to 1 that

$$
\frac{\log \gamma}{\log v}<\frac{3+2 r-\sqrt{(3+2 r)^{2}-8}}{4} .
$$

The proof is left to the reader. Interpreting this inequality numerically shows that our current center bunching condition is modest in comparison to the bunching conditions in [21]. For example, when the hyperbolic part of the spectrum is quarter pinched

$$
\mu=v^{2}=\frac{1}{4}
$$

then a satisfactory $\gamma$ is

$$
\gamma \geq .91
$$

In particular the dimensions of the stable and unstable bundles no longer are part of the estimates.

Next, we sharpen (8). Choose $\alpha<\gamma$ and $0<\beta<\theta$ so that

$$
v<\alpha^{2+2 / \beta} \text {. }
$$


Then set

$$
\sigma=\frac{v}{\alpha}
$$

Therefore $v<\gamma \sigma$ and we can choose $\tau$ between them,

$$
v<\tau<\gamma \sigma .
$$

From (9) and (11) we deduce

$$
\left(\frac{\sigma}{\gamma}\right)^{1+\beta}<\tau<\frac{\sigma}{\gamma}
$$

For

$$
\left(\frac{\sigma}{\gamma}\right)^{1+\beta}=\nu \frac{\nu^{\beta}}{(\alpha \gamma)^{1+\beta}}<\nu \frac{\nu^{\beta}}{\alpha^{2+2 \beta}}<\nu<\tau<\gamma \sigma<\frac{\sigma}{\gamma} .
$$

See Fig. 4 which summarizes the relations among the constants and exponents.
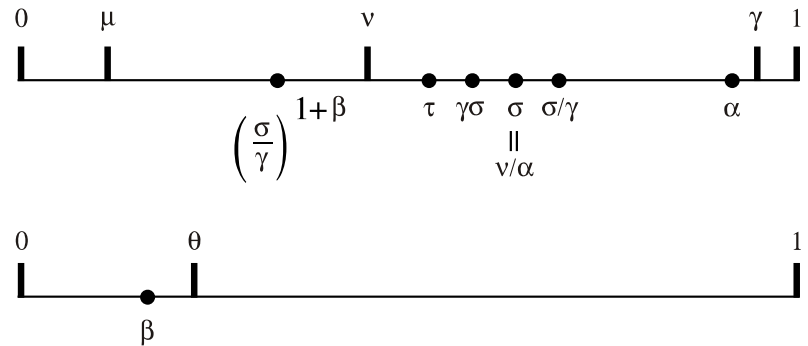

Fig. 4. Constants denoted with a bar are imposed by the diffeomorphism; those denoted with a dot are chosen afterward

Now we fix a point $p \in M$ and specify sequences

$$
\begin{array}{lll}
X_{n}=f^{-n}\left(W^{u}\left(f^{n}(p), \tau^{n}\right)\right) & \subset W_{\mathrm{loc}}^{u}(p) \\
D_{n}=W^{c}\left(p, \sigma^{n}\right) & & \subset W_{\mathrm{loc}}^{c}(p) \\
Y_{n}=f^{n}\left(W^{s}\left(f^{-n}(p), \tau^{n}\right)\right) & \subset W_{\mathrm{loc}}^{s}(p) .
\end{array}
$$

According to the general julienne definition we get sequences of juliennes at $p$

$$
\begin{aligned}
& J_{n}^{c u}=W^{c \cap u}\left(X_{n} \cup D_{n}\right) \\
& J_{n}^{c s}=W^{c \cap s}\left(D_{n} \cup Y_{n}\right) \\
& J_{n}^{\text {solid }}=W^{u \cap s}\left(J_{n}^{c u} \cup J_{n}^{c s}\right),
\end{aligned}
$$


We refer to this $n$ as the rank of the julienne, and to $1 / n$ as its size. As $n \rightarrow \infty$ the juliennes shrink down to $p$.

The collections of these juliennes are denoted

$$
\mathscr{J}_{p}^{c s}=\left\{J_{n}^{c s}: n \in \mathbb{N}\right\} \quad \mathscr{J}_{p}^{c u}=\left\{J_{n}^{c u}: n \in \mathbb{N}\right\} \quad \mathscr{J}_{p}=\left\{J_{n}: n \in \mathbb{N}\right\} .
$$

Letting $p$ range over $M$ gives a solid julienne Vitali basis

$$
\mathscr{J}=\bigcup_{p \in M} \mathscr{T}_{p}
$$

Similarly if $q$ ranges over $W^{c s}(p)$ or $W^{c u}(p)$ we get center stable and center unstable julienne Vitali bases,

$$
\mathscr{J}^{c s}=\bigcup_{q \in W^{c s}(p)} \mathscr{J}_{q}^{c s} \quad \mathscr{J}^{c u}=\bigcup_{q \in W^{c u}(p)} \mathscr{J}_{q}^{c u}
$$

for the leaves $W^{c s}(p), W^{c u}(p)$.

Theorem 4.2. $\mathscr{J}$ is volumetricallyengulfing with respect to Riemann measure on $M ; \mathscr{J}^{c s}$ and $\mathscr{J}^{c u}$ are volumetrically engulfing with respect to Riemann measure on $W^{c s}(p)$ and $W^{c u}(p)$.

Corollary 4.3. The julienne Vitali bases are density bases: almost every point of a measurable set is a julienne density point.

Proof. This a direct application of Theorem 3.1, which states that a volumetrically engulfing Vitali basis is a density basis.

Theorem 4.4. The unstable and stable holonomy maps are julienne quasiconformal: the unstable holonomy is quasi-conformal with respect to the Vitali basis $\mathscr{J}^{c s}$, while the stable holonomy is quasi-conformal with respect to $\mathscr{J}^{\text {cu }}$.

The proofs of Theorems 4.2 and 4.4 appear in Sects. 6, 7, and 8.

\section{Julienne shape}

In this section we study the shape of center stable juliennes. Everything we say has its counterpart for center unstable juliennes. A center stable julienne is a foliation product

$$
J_{n}^{c s}=W^{c \cap s}\left(D_{n} \cup Y_{n}\right)
$$

where $D_{n}$ is small round disc in $W^{c}(p)$ and $Y_{n}$ is a small set in $W^{s}(p)$. It is unclear, a priori, what a julienne looks like, beyond the fact that it is small. For although $f^{-n}\left(Y_{n}\right)=W^{s}\left(f^{-n}(p), \tau^{n}\right)$ is round, there is no reason to expect that its $f^{n}$ image $Y_{n}$ is round or quasi-round. The same 
applies to the pre-julienne $f^{-n}\left(J_{n}^{c s}\right)$. It is the foliation product of a small set $f^{-n}\left(D_{n}\right) \subset W^{c}\left(f^{-n}(p)\right)$ and a round $\operatorname{disc} f^{-n}\left(Y_{n}\right)=W^{s}\left(f^{-n}(p), \tau^{n}\right)$. The julienne and pre-julienne each have one round factor and one non-round factor.

A set that can be expressed as

$$
T=\bigcup_{x \in B} W^{s}(x, r)
$$

for some $B \subset W_{\text {loc }}^{c}(p)$ and some $r>0$ is a center stable tube

$$
T=T^{c s}(B, r)
$$

with base $B$ and stable radius $r$. The stable fibers of a tube are by definition round. The shape of its base can be arbitrary. See Figs. 5, 6 .

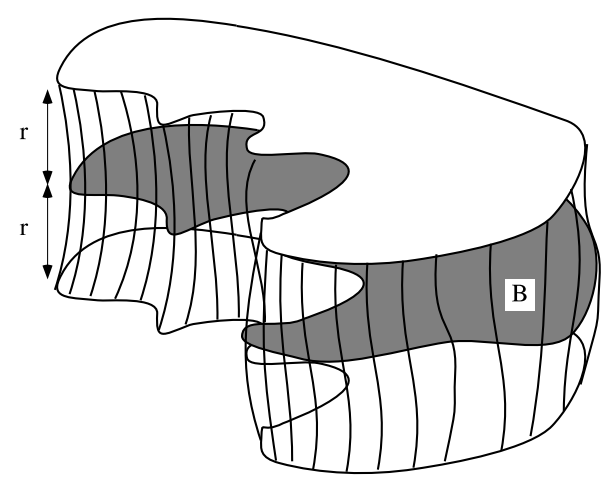

Fig. 5. A center stable tube with one dimensional stable fibers and two dimensional base

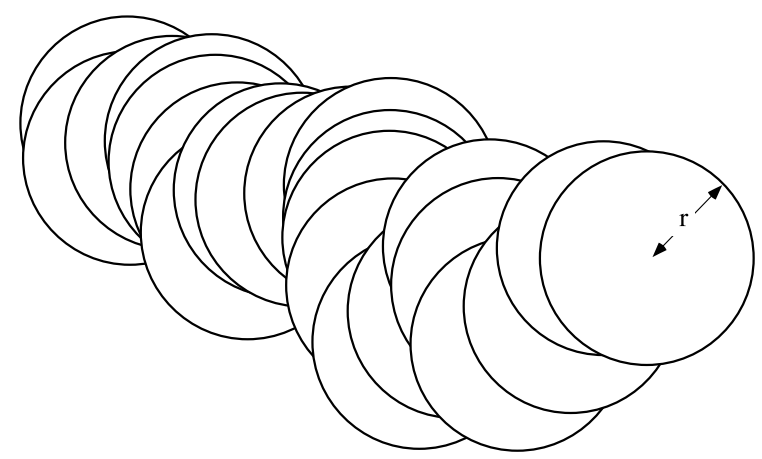

Fig. 6. A center stable tube with two dimensional stable fibers and one dimensional base 
Proposition 5.1. The pre-julienne $f^{-n}\left(J_{n}^{c s}\right)$ is tube-like. It consists of the center core $f^{-n}\left(D_{n}\right)$, through which pass s-fibers. The s-fibers are approximately round discs of radius $\tau^{n}$. More precisely, given $\epsilon>0$, if $n$ is large, then the pre-julienne is bracketted between tubes with base $f^{-n}\left(D_{n}\right)$ and stable radii $(1 \pm \epsilon) \tau^{n}$.

The proof of this proposition involves the center bunching and Hölder assumptions used to choose the julienne shape. The next lemma generalizes easily to any foliation whose tangent bundle is $\theta$-Hölder continuous, where $0<\beta<\theta \leq 1$.

Lemma 5.2. The center holonomy $h: W^{s}(p) \rightarrow W^{s}(q), h(p)=q$, is approximately isometric at small $\beta$-Hölder scale in the following sense. Given $\epsilon>0$ there is a $\delta>0$ such that if

$$
r^{1+\beta} \leq \rho \leq r<\delta
$$

and if $q \in W^{c}(p, r)$, then $h$ carries a round disc $D$ of radius $\rho$ in $W^{s}(p)$ to a nearly round disc $h(D)$ of radius $\rho$ in $W^{s}(q)$. More precisely, if $D=$ $W^{s}(p, \rho)$ then $h(D)$ is bracketted between round discs

$$
W^{s}(q,(1-\epsilon) \rho) \subset h(D) \subset W^{s}(q,(1+\epsilon) \rho) .
$$

Proof. The point to note here is that $\rho$ can be quite a bit smaller than $r$, but not arbitrarily smaller; it can never be less than $r^{2}$. Discs whose radii are too small may be grossly distorted in shape by the center holonomy map $h$. See Fig. 7, which indicates that the shape and size of $h(D)$ is under good control at one scale, but still may be pathological at a smaller scale. We recapitulate

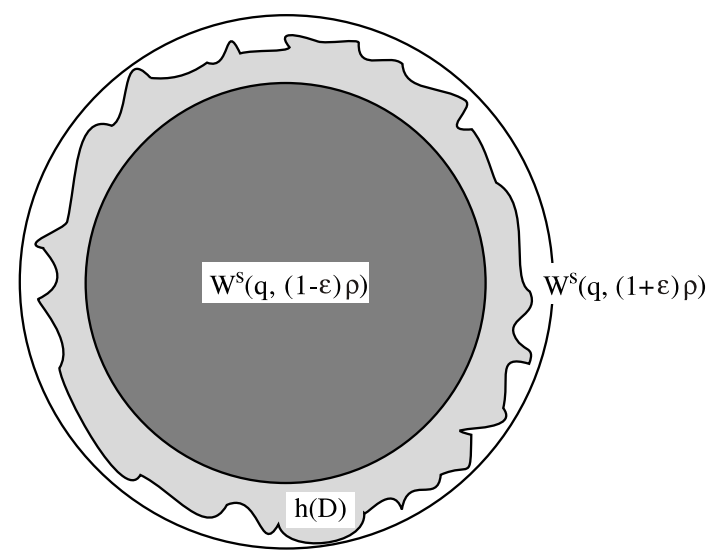

Fig. 7. At small $\beta$-Hölder scale $h(D)$ is approximately round 
the proofs of Lemmas 1 and 2 in Sect. 4 of [21]. In an exponential chart at $p$, the center leaf through a point $z$ near $p$ is the graph of a function

$$
\phi_{z}: E_{p}^{c}(r) \rightarrow E_{p}^{s} .
$$

See Fig. 8. The function $\phi_{z}(x)$ is differentiable with respect to $x$. The norm

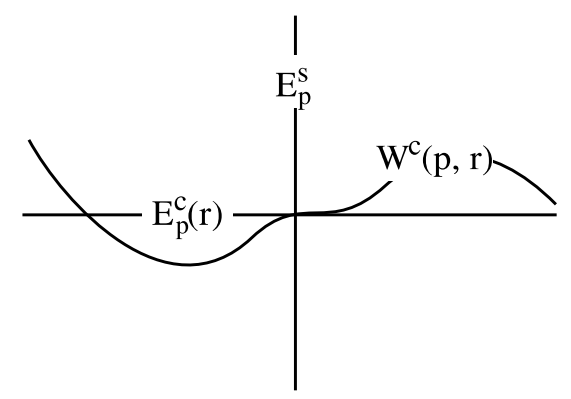

Fig. 8. Locally, in exponential charts, leaves are graphs

of its derivative $\partial \phi_{z} / \partial x$ is the slope of the leaf in the exponential chart. By assumption $\partial \phi_{z} / \partial x$ is $\theta$-Hölder continuous, and at the point $(z, x)=(p, 0)$, $\partial \phi_{z} / \partial x=0$. Thus

$$
\left\|\frac{\partial \phi_{z}}{\partial x}\right\| \leq K r^{\theta}
$$

where $(z, x)$ varies in the $r$-neighborhood of $(p, 0)$. Geometrically, this means that the center leaves are quite flat, quite horizontal.

Consider the horizontal holonomy $H$. It is translation parallel to the plane $E_{p}^{c}$. Continuity of $E^{s}$ implies that $H$ has the following property. Given $\epsilon>0$ there is a $\delta>0$ such that if $\rho \leq r<\delta$ then

$$
H(D) \subset W^{s}(H p,(1+\epsilon / 2) \rho) .
$$

See Fig. 9. No underbound for $\rho$ is needed here.

Over a distance $r$, the center leaf diverges from the horizontal plane parallel to $E_{p}^{c}$ by at most

$$
K r^{\theta} r=K r^{1+\theta},
$$

a quantity that is negligible in comparison to $\rho$. For when $r$ is small, our choice in (9) of $\beta<\theta$ and (13) imply that

$$
r^{1+\theta} \ll r^{1+\beta} \leq \rho
$$

Hence, $|H p-q| \ll \rho$ and

$$
h(D) \subset W^{s}(q,(1+\epsilon) \rho) .
$$




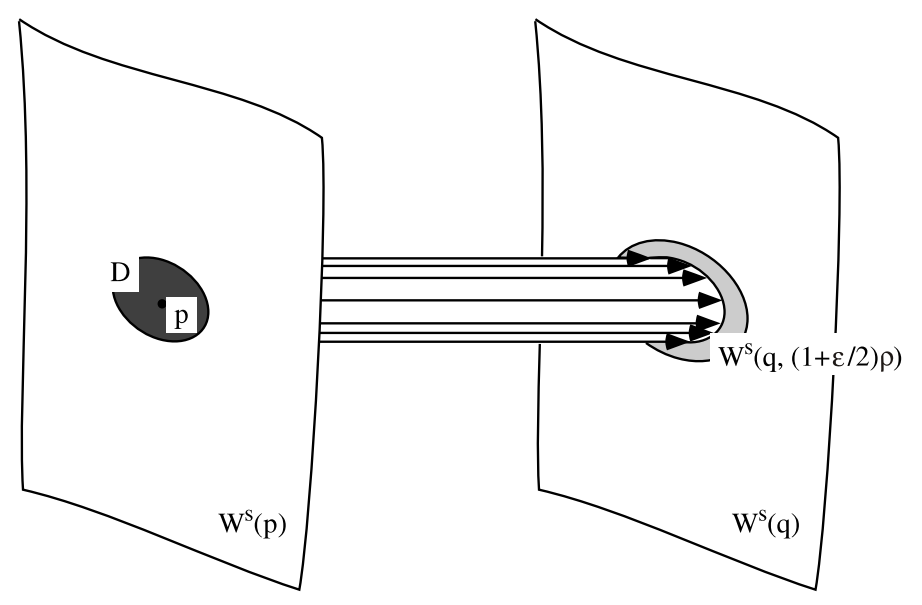

Fig. 9. At small scale, the horizontal holonomy is approximately isometric

Applying the same reasoning to $h^{-1}$, we see that

$$
h^{-1}\left(W^{s}(q,(1-\epsilon) \rho)\right) \subset D,
$$

and it follows that $h(D)$ is bracketted between the round discs $W^{s}(q,(1 \pm \epsilon) \rho)$ as claimed.

Proof of Proposition 5.1. The main stable fiber of the pre-julienne

$$
f^{-n}\left(J_{n}^{c s}\right)=W^{c \cap s}\left(f^{-n}\left(D_{n}\right) \cup W^{s}\left(f^{-n}(p), \tau^{n}\right)\right)
$$

is the round disc $W^{s}\left(f^{-n}(p), \tau^{n}\right)$, while the center core is $f^{-n}\left(D_{n}\right)=$ $f^{-n}\left(W^{c}\left(p, \sigma^{n}\right)\right)$. Since $\left\|T^{c} f\right\| \leq \gamma^{-1}$,

$$
f^{-n}\left(W^{c}\left(p, \sigma^{n}\right)\right) \subset W^{c}\left(f^{-n}(p), \sigma^{n} / \gamma^{n}\right) .
$$

We will apply Lemma 5.2, with $r=\sigma^{n} / \gamma^{n}$ and $\rho=\tau^{n}$. We need to check (13), namely,

$$
\left(\frac{\sigma^{n}}{\gamma^{n}}\right)^{1+\beta}<\tau^{n}<\frac{\sigma^{n}}{\gamma^{n}},
$$

and by (12), this is true. Also, when $n$ is large, $r=\sigma^{n} / \gamma^{n}$ is small. Lemma 5.2 then completes the proof.

\section{Juliennes under unit holonomy}

In this section we study the unstable holonomy $h: W^{c s}(p) \rightarrow W^{c s}(q)$, $q=h(p)$, and how it affects center stable juliennes. Everything we say has its counterpart for center unstable juliennes. We assume throughout that $q$ lies in the unit unstable manifold of $p$,

$$
q \in W^{u}(p, 1) .
$$


Theorem 6.1. $h$ disrupts rank in a uniformly bounded fashion: there is an integer $k$ such that for all $p \in M$ and all large $n$,

$$
J_{n+k}^{c s}(q) \subset h\left(J_{n}^{c s}(p)\right) \subset J_{n-k}^{c s}(q) .
$$

Proof. The subtlety of the analysis is present exactly here. The shapes of the juliennes may be awful, but in terms of holonomy they nest.

At the end of the proof we show that index juggling reduces (14) to

$$
h\left(J_{n+k}^{c s}(p)\right) \subset J_{n}^{c s}(q) .
$$

Referring to $J_{n+k}^{c s}(p)$ as the small julienne at $p$ and to $J_{n}^{c s}(q)$ as the large julienne at $q,(15)$ asserts that holonomy carries the small julienne into the large one.

First we determine $k$. The unstable holonomy map $h$ sends center manifolds to center manifolds, and its restriction to each individual center manifold is of class $C^{1}$ or better. See Theorem B of [23]. (On the full center stable manifold $h$ is of course much less regular than Lipschitz.) By compactness, the supremum, $L(h) \geq 1$, of the Lipschitz constants of all the local unstable holonomy maps $h: W^{c}(p) \rightarrow W^{c}(q)$ is finite. Choose $k$ such that

$$
\sigma^{k} \leq \frac{1}{6 L(h)} .
$$

The center core disc $D_{n+k}(p)$ has radius $\sigma^{n+k}$, and thus $h$ sends it to a subset of $W^{c}(q)$ having radius $L(h) \sigma^{n+k}$, which by the choice of $k$ in (16) is less than $\sigma^{n} / 2$. Thus

$$
h\left(D_{n+k}(p)\right) \subset \frac{1}{2} D_{n}(q),
$$

where $\frac{1}{2} D_{n}(q)$ is the half-sized sub-disc of $D_{n}(q)$. The inclusion (17) is a step toward (15). We want to show that the holonomy image of one julienne is contained in another julienne, and (17) shows that at least this is true of their center cores.

Since the unstable holonomy is defined by the invariant foliation $\mathscr{T}^{u}$, it commutes with $f$. Specifically, if we write

$$
h_{n}: W^{c s}\left(f^{-n}(p)\right) \rightarrow W^{c s}\left(f^{-n}(q)\right)
$$

then

$$
h=h_{0}=f^{n} \circ h_{n} \circ f^{-n} .
$$

Thus, (15) is equivalent to

$$
h_{n} \circ f^{-n}\left(J_{n+k}^{c s}(p)\right) \subset f^{-n}\left(J_{n}^{c s}(q)\right) .
$$

Think of the holonomy map as taking an object located on one center stable manifold, and "pushing it across" to another center stable manifold. Then (18) has two advantages over (15). 
(a) The distance between the domain and target for $h_{n}$ (the "push across distance") is much less than that for $h$.

(b) The domain and target of $h_{n}$ in (18) are more round in shape than those that confront $h$ in (15). For pre-juliennes are less elongated than juliennes.

To prove (18) it suffices to find tubes $T_{n}, T_{n}^{\prime}$ such that

$$
\begin{aligned}
f^{-n}\left(J_{n+k}^{c s}(p)\right) \subset & T_{n} \\
& h_{n}\left(T_{n}\right) \subset T_{n}^{\prime} \subset f^{-n}\left(J_{n}^{c s}(q)\right) .
\end{aligned}
$$

Set

$$
B_{n}=f^{-n}\left(\frac{1}{2} D_{n}(q)\right) \quad B_{n}^{\prime}=f^{-n}\left(D_{n}(q)\right) .
$$

By Proposition 5.1 the pre-julienne $f^{-n}\left(J_{n}^{c s}(q)\right)$ is tube like. It contains a tube $T_{n}^{\prime}$ with base $B_{n}^{\prime}$ and stable radius $2 \tau^{n} / 3$. The distance from $\frac{1}{2} D_{n}(q)$ to the boundary of $D_{n}(q)$ is $\sigma^{n} / 2$. Under $f^{-n}$ this distance can decrease by at worst the factor $\gamma^{n}$. Thus,

$$
x \in B_{n} \Rightarrow d\left(x, \partial B_{n}^{\prime}\right) \geq \frac{(\gamma \sigma)^{n}}{2} .
$$

See Fig. 10. We do not assert that $B_{n}$ lies in the middle of $B_{n}^{\prime}$, merely that its distance to $\partial B_{n}^{\prime}$ decays no faster than a slow exponential. The shape of the pair $B_{n} \subset B_{n}^{\prime}$ can in principle be quite messy.

By Proposition 5.1 the pre-julienne $f^{-(n+k)}\left(J_{n+k}^{c s}(p)\right)$ is also tube-like. It is contained in a tube $T_{n+k}$,

$$
f^{-(n+k)}\left(J_{n+k}^{c s}(p)\right) \subset T_{n+k}=\bigcup_{x \in f^{-(n+k)}\left(D_{n+k}(p)\right)} W^{s}\left(x, 2 \tau^{n+k}\right) .
$$

Since $\left\|T^{s} f\right\| \leq v<\sigma$, we see from (16) that

$$
f^{-n}\left(J_{n+k}^{c s}(p)\right) \subset f^{k}\left(T_{n+k}\right) \subset \bigcup_{x \in f^{-n}\left(D_{n+k}(p)\right)} W^{s}\left(x, \tau^{n} / 3\right)=T_{n},
$$

a tube in $W^{c s}\left(f^{-n}(p)\right)$ with base $f^{-n}\left(D_{n+k}(p)\right)$ and stable radius $\tau^{n} / 3$.

We claim that $h_{n}\left(T_{n}\right) \subset T_{n}^{\prime}$. Take a point $x \in T_{n}$ that lies in $f^{-n}\left(D_{n+k}(p)\right)$. Since $h_{n} \circ f^{-n}=f^{-n} \circ h$, (17) implies that

$$
h_{n}(x) \in f^{-n}\left(\frac{1}{2} D_{n}(q)\right)=B_{n} .
$$

Take a point $y \in T_{n}$ that lies in $W^{s}\left(x, \tau^{n} / 3\right)$. The distance between the center stable manifolds through $f^{-n}(p)$ and $f^{-n}(q)$ is at most $v^{n}$ since their original distance apart is at most 1 , and $\left\|T^{u} f^{-1}\right\| \leq v$. The points $x, y$ lie on the former and $h_{n}(x), h_{n}(y)$ lie on the latter. By the triangle inequality,

$$
d\left(h_{n}(x), h_{n}(y)\right) \leq d\left(h_{n}(x), x\right)+d(x, y)+d\left(y, h_{n}(y)\right) \leq 2 v^{n}+\tau^{n} / 3 .
$$




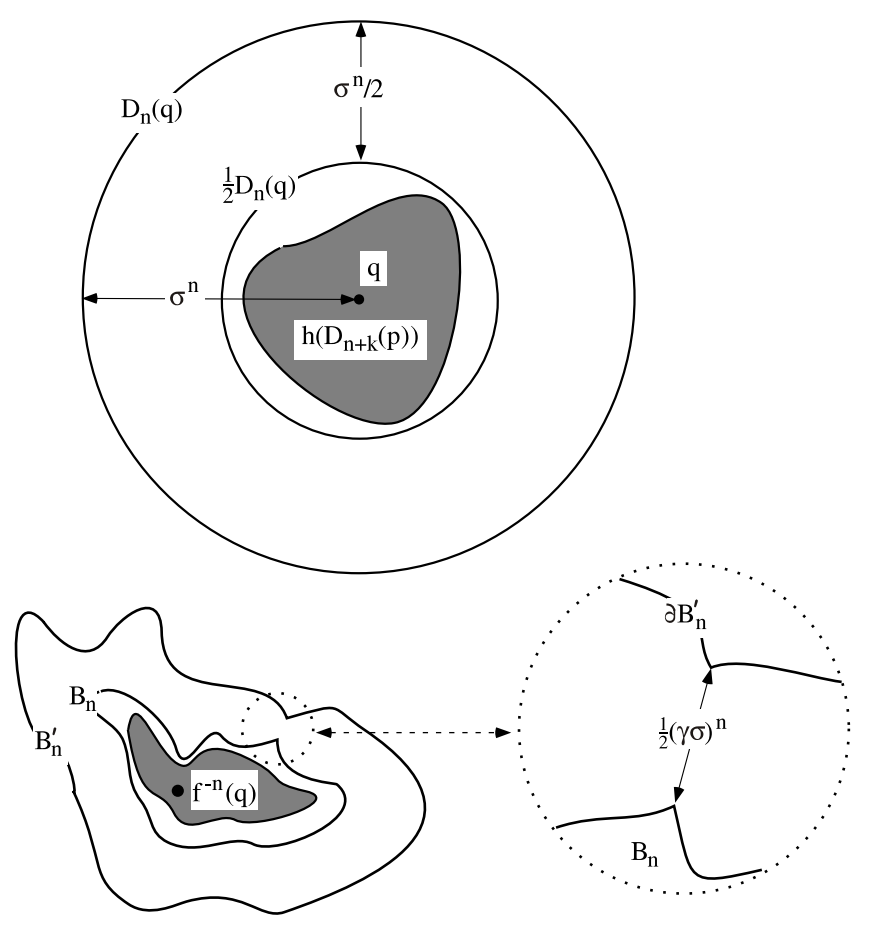

Fig. 10. The center cores of the julienne and pre-julienne at $q$ and at $f^{-n}(q)$

Since $v<\tau<\gamma \sigma$, for large $n$ we have

$$
d\left(h_{n}(x), h_{n}(y)\right) \leq \tau^{n} / 2 \ll \operatorname{dist}\left(B_{n}, \partial B_{n}^{\prime}\right) .
$$

Although potentially ugly themselves, the sets $B_{n}, B_{n}^{\prime}$ are contained in $W^{c}=W_{\text {loc }}^{c}\left(f^{-n}(q)\right)$, which is a round disc. The point $h_{n}(y)$ is not near the boundary of $W^{c}$ because it much nearer the point $h_{n}(x) \in B_{n}$ than the distance between $B_{n}$ and $\partial B_{n}^{\prime}$. The shortest distance from $h_{n}(y)$ to $W^{c}$ is essentially achieved along the local stable manifolds, so the distance from $h_{n}(y)$ to $W^{c}$ is essentially $\leq \tau^{n} / 2$, and we get an intersection point

$$
z \in W^{s}\left(h_{n}(y), \tau^{n} / 2\right) \cap W^{c} .
$$

Then $d(z, x) \leq \tau^{n} \ll \operatorname{dist}\left(B_{n}, \partial B_{n}^{\prime}\right)$ implies that $z \in B_{n}^{\prime}$. In other words, $h_{n}(y) \in W^{s}\left(B_{n}^{\prime}, \tau^{n} / 2\right)$, which is contained in the tube $T_{n}^{\prime}$. See Fig. 11 . This implies (19), hence (18), and hence (15).

It remains to deduce (14) from (15). Since the latter is valid for all large $n$, we can replace $n$ with $n-k$. This gives half of (14),

$$
h\left(J_{n}^{c s}(p)\right) \subset J_{n-k}^{c s}(q) .
$$




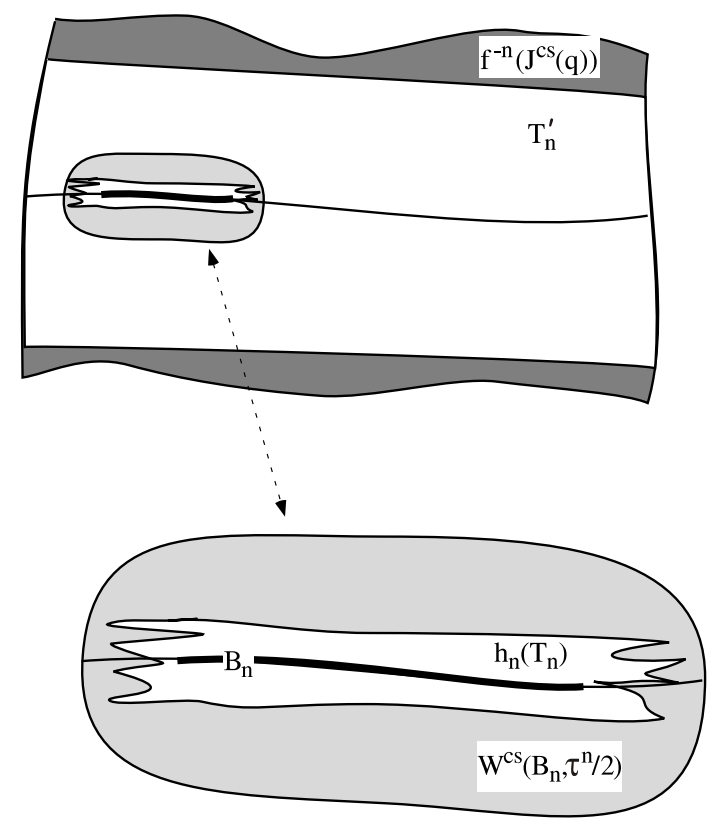

Fig. 11. Despite its ragged ends, $h_{n}\left(T_{n}\right)$ is contained in $T_{n}^{\prime}$

Interchanging $p$ and $q$, and applying (15) to $h^{-1}$ instead of to $h$, we get

$$
h^{-1}\left(J_{n+k}^{c s}(q)\right) \subset J_{n}^{c s}(p) .
$$

Applying $h$ to both sides of (21), we get the other half of (14).

\section{Juliennes engulf}

In this section we verify the geometric part of the volumetric engulfing property. In the next section we work out the measure ratio estimate.

Theorem 7.1. Center stable juliennes have the following engulfing property. There is an $\ell \in \mathbb{N}$ such that if two center stable juliennes $J_{n+\ell}^{c s}, J_{n+\ell}^{c s}{ }^{\prime}$ meet then

$$
J_{n+\ell}^{c s} \subset J_{n}^{c s} .
$$

Proof. Recall that $n$ is the rank of the julienne $J_{n}^{c s}$. The theorem asserts that a rank $n$ julienne will engulf a rank $n+\ell$ julienne, if the latter meets the 
rank $n+\ell$ sub-julienne of the former. Write

$$
\begin{aligned}
& J_{n}^{c s}=W^{c \cap s}\left(D_{n} \cup Y_{n}\right) \\
& J_{n+\ell}^{c s}=W^{c \cap s}\left(D_{n+\ell} \cup Y_{n+\ell}\right) \\
& J_{n+\ell}^{c s}{ }^{\prime}=W^{c \cap s}\left(D_{n+\ell}^{\prime} \cup Y_{n+\ell}^{\prime}\right) .
\end{aligned}
$$

We refer to these juliennes as the big julienne, the small julienne, and the neighboring julienne, respectively. According to Proposition 5.1, the stable fibers of a julienne of rank $n$ are images by $f^{n}$ of approximately round discs of radius $\tau^{n}$. Since $\left\|T^{s} f\right\| \leq \nu$, these $f^{n}$ images are contained in stable discs of radius $v^{n} \tau^{n}<v^{n} \sigma^{n}$, a number that is much smaller than the radius $\sigma^{n}$ of the core disc $D_{n}$. Thus, the diameter of a center stable julienne is approximately the diameter of its core disc, and the diameter of the neighboring julienne that we hope to engulf is approximately $\sigma^{n+\ell}$. We choose $\ell$ so that

$$
\sigma^{\ell} \leq \frac{1}{3}
$$

Then the the two intersecting juliennes of rank $n+\ell$ have diameter less than $1 / 3$ of the diameter of the big julienne, and one of them, $J_{n+\ell}^{c s}$, includes the point $p$. It follows that the neighboring julienne, $J_{n+\ell}^{c s}$, is so close to the middle of the center core disc $D_{n}$, of the big julienne that it projects into $D_{n}$ under the stable holonomy map. That is

$$
J_{n+\ell}^{c s} \subset W_{\text {loc }}^{s}\left(J_{n}^{c s}\right) .
$$

See Fig. 12. All the $s$-fibers of the neighboring julienne lie on local stable manifolds passing through the core disc $D_{n}$ of the big julienne. If we can show that all the $c$-fibers of the neighboring julienne lie on local center manifolds passing through the main stable fiber $Y_{n}$ of the big julienne, then

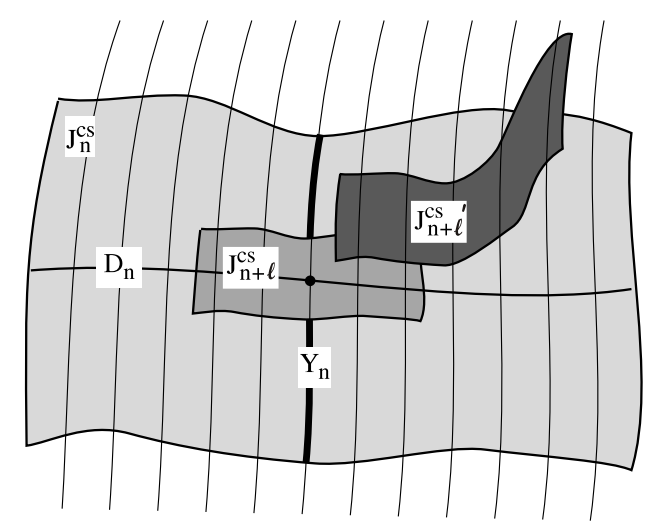

Fig. 12. The neighboring julienne is contained in the local stable saturate of $D_{n}$ 
by the naturality of the julienne definition, Proposition 4.1, the big julienne engulfs the neighboring julienne.

To understand the center fibers, we apply $f^{-n}$ to the picture. According to Proposition 5.1 the big julienne becomes tube-like. We refer to the $f^{-n}$ images of the other two juliennes as the small pre-julienne and the neighboring pre-julienne. Their $s$-fibers, being the $f^{\ell}$-images of approximately round discs of radius $\tau^{n+\ell}$, are contained in round $s$-discs of radius $\nu^{\ell} \tau^{n+\ell}$. Moreover, the center core of the small pre-julienne, $f^{-n}\left(D_{n+\ell}\right)$, is contained in the center core $f^{-n}\left(D_{n}\right)$ of the tube-like pre-julienne, and its stable fibers are contained in discs, centered at points of $f^{-n}\left(D_{n}\right)$, that have radius $<\tau^{n} / 3$. One of these $s$-fibers of the small pre-julienne meets an $s$-fiber of the neighboring pre-julienne. The latter $s$-fiber lies in a disc of radius $<\tau^{n} / 3$. Two intersecting discs of these sizes and positions are contained in the $s$-disc of radius $\tau^{n}$. See Fig. 13.

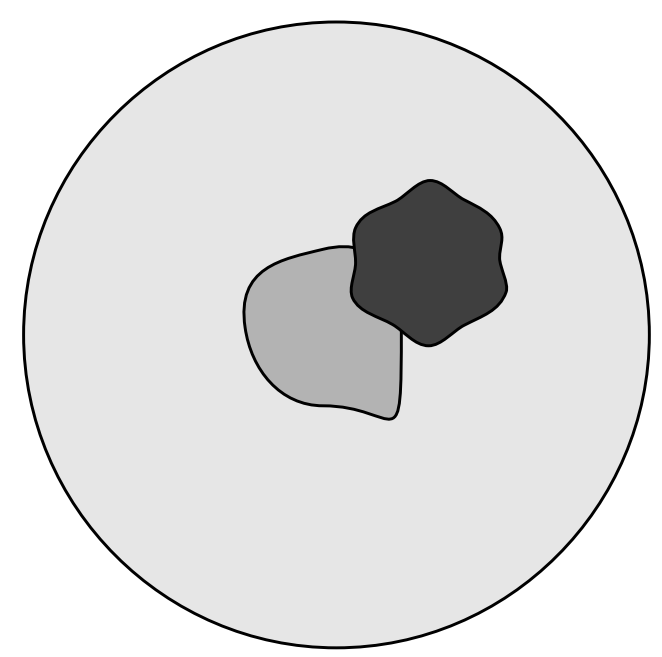

Fig. 13. If a small, centered sub-disc of a big disc meets a second small disc, the big disc engulfs it

Hence, at least one, special $s$-fiber of the neighboring pre-julienne is contained in an $s$-fiber of the big, tube-like pre-julienne. By the construction of juliennes as foliation products, all points on an $s$-fiber of a julienne lie on local center manifolds that pass through the main stable fiber of the julienne. See Proposition 4.1. Therefore, all points of the special $s$-fiber of the neighboring pre-julienne lie both on local center manifolds that pass through the main stable fiber of the big pre-julienne, and on local center manifolds that pass through the main stable fiber of the neighboring prejulienne. Local center manifolds are unique. Therefore, all the $c$-fibers of 
the neighboring pre-julienne lie on local center manifolds passing through the main stable fiber $f^{-n}\left(Y_{n}\right)$ of the big pre-julienne. Re-applying $f^{n}$, we conclude that the same is true of the juliennes themselves: all the $c$-fibers of the neighboring julienne lie on local center manifolds passing through the main stable fiber $Y_{n}$ of the big julienne. This implies that the big julienne engulfs the neighboring julienne.

Theorem 7.2. Solidjuliennes have the same engulfing property: there exists an integer $L$ such that a rank $n$ julienne will engulf a rank $n+L$ julienne, if the latter meets the rank $n+L$ sub-julienne of the former.

Lemma 7.3. If $J, J^{\prime}$ are solid juliennes and the local unstable and local stable saturates of $J$ contain the main center stable and center unstable slices of $J^{\prime}$,

$$
W_{\mathrm{loc}}^{u}(J) \supset J^{c s^{\prime}} \text { and } W_{\mathrm{loc}}^{s}(J) \supset J^{c u \prime} \text {, }
$$

then $J \supset J^{\prime}$.

Proof. The proof is like that of Proposition 4.1. Take a point $z^{\prime} \in J^{\prime}$. It is the intersection point for a pair of local unstable and stable manifolds,

$$
z^{\prime}=W_{\mathrm{loc}}^{s}\left(x^{\prime}\right) \cap W_{\mathrm{loc}}^{u}\left(y^{\prime}\right)
$$

where $x^{\prime} \in J^{c u^{\prime}}, y^{\prime} \in J^{c s^{\prime}}$. But by assumption, $x^{\prime}$ lies on a local stable manifold through the main center unstable slice $J^{c u}$ of $J$, and $y^{\prime}$ lies on a local unstable manifold through the main center stable slice $J^{c s}$ of $J$, say

$$
x^{\prime} \in W_{\mathrm{loc}}^{s}(x) \quad y^{\prime} \in W_{\mathrm{loc}}^{u}(y)
$$

for some $x \in J^{c u}, y \in J^{c s}$. Since local stable and unstable manifolds are unique, we see that $z^{\prime} \in J$.

Proof of Theorem 7.2. Theorems 6.1 and 7.1 produce integers $k, \ell$. The first expresses the rank disruption caused by local holonomy, while the second expresses the center stable and center unstable rank disruption caused by neighborly engulfing. We claim that $L=2 \ell+k$ expresses the rank disruption caused by solid neighborly engulfing.

Suppose that $J_{n}(p), J_{n}\left(p^{\prime}\right)$ are two rank $n$ solid juliennes that meet at a point $z$. By the julienne construction, $z$ lies on a local unstable manifold through the main center stable slice $J_{n}^{c s}$ of $J_{n}$, and also on a local unstable manifold through the main center stable slice $J_{n}^{c s^{\prime}}$ of $J_{n}^{\prime}$. Local unstable manifolds are unique, so the local unstable manifold through $z$ passes through both $J_{n}^{c s}$ and $J_{n}^{c s \prime}$, say at $y$ and $y^{\prime}$. See Fig. 14. Dually, the local stable manifold through $z$ passes through the main center unstable slices of both $J_{n}$ and $J_{n}^{\prime}$. 


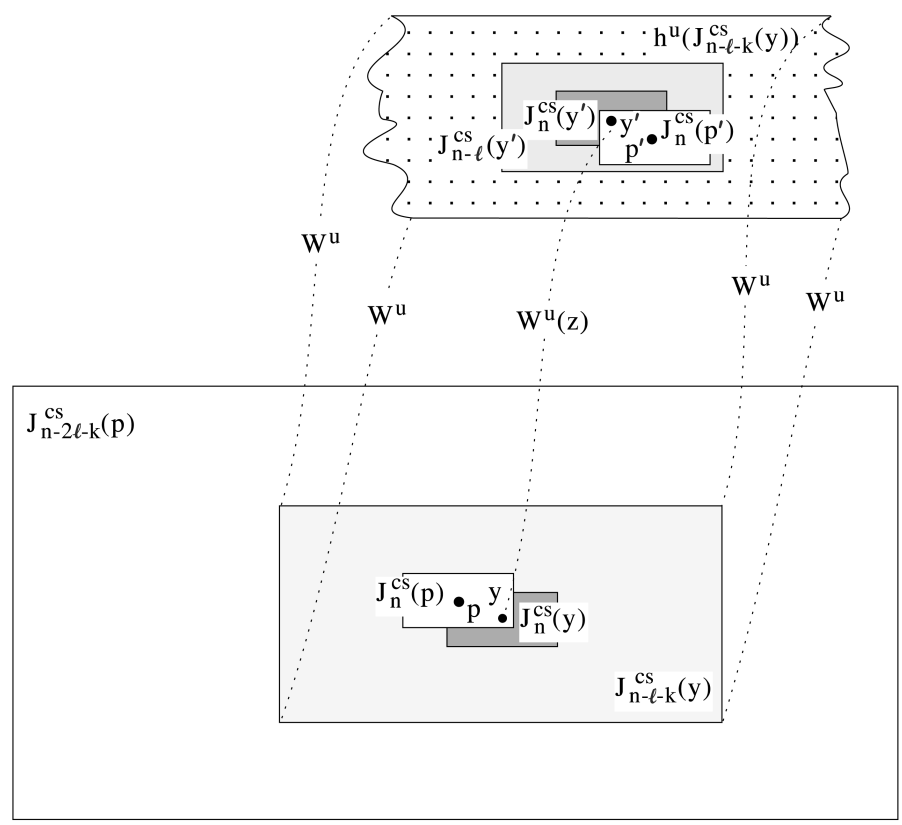

Fig. 14. The centers are horizontal, the stables are vertical, and the unstables retreat into the page obliquely. The white rectangles are center stable juliennes at $p$ and $p^{\prime}$. The shaded rectangles are center stable juliennes at $y$ and $y^{\prime}$. The dotted lattice region is the holonomy image of $J_{n-\ell-k}^{c s}(y)$. Thus

$$
J_{n}^{c s}\left(p^{\prime}\right) \subset J_{n-\ell}^{c s}\left(y^{\prime}\right) \subset h^{u}\left(J_{n-\ell-k}^{c s}(y)\right) \subset W_{\mathrm{loc}}^{u}\left(J_{n-2 \ell-k}^{c s}(p)\right)
$$

We apply Theorem 7.1 to the rank $n$ center stable julienne $J_{n}^{c s}\left(y^{\prime}\right)$ at $y^{\prime}$. Decreasing its rank by $\ell$ causes it to engulf $J_{n}^{c s}\left(p^{\prime}\right)$,

$$
J_{n-\ell}^{c s}\left(y^{\prime}\right) \supset J_{n}^{c s}\left(p^{\prime}\right) .
$$

Then we apply Theorem 6.1 to the center stable julienne $J_{n-\ell}^{c s}(y)$ at $y$. Decreasing its rank by $k$ causes its unstable holonomy image to engulf $J_{n-\ell}^{c s}\left(y^{\prime}\right)$,

$$
h^{u}\left(J_{n-\ell-k}^{c s}(y)\right) \supset J_{n-\ell}^{c s}\left(y^{\prime}\right) .
$$

Finally, decreasing the rank of the center stable julienne $J_{n-\ell-k}^{c s}(p)$ by a further $\ell$ causes it to engulf $J_{n-\ell-k}^{c s}(y)$,

$$
J_{n-2 \ell-k}^{c s}(p) \supset J_{n-\ell-k}^{c s}(y) .
$$

Combining (22), (23), and (24), we see that the unstable holonomy image of $J_{n-2 \ell-k}^{c s}(p)$ engulfs $J_{n}^{c s}\left(p^{\prime}\right)$. In terms of saturates this means

$$
W_{\mathrm{loc}}^{u}\left(J_{n-2 \ell-k}^{c s}(p)\right) \supset J_{n}^{c s}\left(p^{\prime}\right) .
$$


Dually we see that

$$
W_{\mathrm{loc}}^{s}\left(J_{n-2 \ell-k}^{c u}(p)\right) \supset J_{n}^{c u}\left(p^{\prime}\right) .
$$

Then, according to Lemma 7.3, $J \supset J^{\prime}$.

We conclude this section with an extension of Proposition 5.1. A set that can be expressed as

$$
T=\bigcup_{x \in B} W^{s}(x, r)
$$

for some $B \subset W_{\text {loc }}^{c u}(p)$ and some $r>0$ is a solid tube

$$
T=T^{\text {solid }}(B, r)
$$

with base $B$ and stable radius $r$.

Theorem 7.4. Let $L$ be the rank disruption bound produced in Theorem 7.2. A solid pre-julienne $f^{-n}\left(J_{n}(p)\right)$ can be bracketted between solid tubes

$$
T_{n} \subset f^{-n}\left(J_{n}(p)\right) \subset T_{n}^{\prime}
$$

such that $T_{n}$ has base $f^{-n}\left(J_{n+L}^{c u}\right)$ and stable radius $r_{n}=(\mu \tau)^{L} \tau^{n}$, while $T_{n}^{\prime}$ has base $f^{-n}\left(J_{n-L}^{c u}\right)$ and stable radius $r_{n}^{\prime}=(\mu \tau)^{-L} \tau^{n}$.

Proof. Theorem 7.2 implies that $J_{n}(p)$ engulfs $J_{n+L}(x)$ if $x \in J_{n+L}^{c u}(p)$. The main stable fiber of $f^{-(n+L)}\left(J_{n+L}^{c s}(x)\right)$ is the round, stable disc $W^{s}\left(f^{-(n+L)}(x), \tau^{n+L}\right)$. The $f^{L}$ image of this disc contains a round, stable sub-disc of radius $r_{n}$. Letting $x$ range over $J_{n+L}^{c u}(p)$, we see that $f^{-n}\left(J_{n}(p)\right)$ contains the tube $T_{n}$. The proof that $f^{-n}\left(J_{n}(p)\right)$ is contained in the tube $T_{n}^{\prime}$ is similar.

\section{Julienne measure}

A Riemann structure on $T M$ that expresses the partial hyperbolicity of our diffeomorphism $f$ is adapted to $f$. Fix such an adapted Riemann structure. It induces Riemann structures tangent to the leaves of the foliations $\mathscr{W}^{u}$, $\mathscr{W}^{c u}, \mathscr{W}^{c}, \mathscr{W}^{c s}, \mathscr{W}^{s}$. These induced Riemann structures give rise to Riemann measures $m, m^{u}, \ldots, m^{s}$ on $M$ and on the leaves of the foliations $\mathscr{W}^{u}, \ldots, \mathscr{W}^{s}$.

We need to estimate the volume of discs and tubes with respect to these measures. To do so, we first modify the exponential charts $\exp _{p}$ : $T_{p} M(\rho) \rightarrow M$ as follows. (Everything will be done locally -i.e., in a modest sized neighborhood of the zero section, say of radius $\rho$.) The exponential pre-image of the local center unstable manifold $W_{\text {loc }}^{c u}(p)$ is a graph,

$$
\exp _{p}^{-1}\left(W_{\mathrm{loc}}^{c u}(p)\right)=\operatorname{graph}\left(\phi_{p}^{c u}\right)
$$


where $\phi_{p}^{c u}: E_{p}^{c u}(\rho) \rightarrow E_{p}^{s}(\rho)$ is a function of at least class $C^{1+\text { Hölder }}$. Write $x \in E_{p}^{c u}(\rho), y \in E_{p}^{s}(\rho)$, and define

$$
\Phi_{p}^{c u}(x, y)=\left(x, y+\phi_{p}^{c u}(x)\right) .
$$

$\Phi_{p}^{c u}$ is a diffeomorphism of $T_{p} M(\rho)$ to another neighborhood of the origin of $T_{p} M$. At the origin of $E_{p}^{c u}, \phi_{p}^{c u}$ and its derivative $D \phi_{p}^{c u}$ vanish. The derivative is taken with respect to $x \in E_{p}^{c u}$. Thus, at the origin of $T_{p} M, D \Phi_{p}^{c u}=I$, the identity map.

The union of all the maps $\Phi_{p}^{c u}$ as $p$ varies in $M$, is a homeomorphism $\Phi^{c u}$ from $T M(\rho)$ to another neighborhood of the zero section. It and its fiber derivative $D \Phi_{p}^{c u}$ are Hölder continuous on $T M$. The composite exp $\circ \Phi^{c u}$ is a partially adapted exponential map. It sends each $E_{p}^{c u}(\rho)$ to $W_{\text {loc }}^{c u}(p)$.

Repeating this type of modification for the other invariant manifolds leads to a homeomorphism $H$ from $T M(\rho)$ to another neighborhood of the zero section covering the identity map on $M$ such that the restriction of $H$ to $T_{p} M(\rho)$ is a $C^{1+\text { Hölder }}$ diffeomorphism which carries the discs $E_{p}^{u}(\rho), E_{p}^{c u}(\rho), E_{p}^{c}(\rho), E_{p}^{c s}(\rho), E_{p}^{s}(\rho)$ to the exponential pre-images of the corresponding local invariant manifolds. The composite

$$
e=\exp \circ H
$$

is the adapted exponential map. Its restriction to $T_{p} M(\rho)$ is a $C^{1+\text { Hölder }}$ local diffeomorphism $e_{p}$ from $T_{p} M(\rho)$ to $M$ sending the discs $E_{p}^{u}(\rho), \ldots$, $E_{p}^{s}(\rho)$ to the corresponding invariant manifolds. Like the unadapted exponential, the tangent to $e_{p}$ at the origin is the identity,

$$
T_{0}\left(e_{p}\right)=I
$$

Using $e$, we lift all objects in $M$ to $T_{p} M$, writing a bar over the lifted object. For example, $S \subset M$ lifts to $\bar{S}=e_{p}^{-1}(S) \subset T_{p} M$. Likewise, the measure $m$ on $M$ lifts to a measure $\bar{m}=e_{p}^{*}(m)$ in $T_{p} M$, the $\bar{m}$-measure of $\bar{S}$ being $m(S)$. The measure $\bar{m}$ is not translation invariant with respect to the linear structure of $T_{p} M$.

The adapted Riemann structure on $T M$ gives each tangent space an inner product, and the inner product induces a second, natural, translation invariant measure, the Riemann measure $m_{p}$ on $T_{p} M$. Due to (25), one can view $m_{p}$ as the linear part of $\bar{m}$ at the origin of $T M_{p}$.

The other measures $m^{u}, \ldots, m^{s}$ lift to measures $\bar{m}^{u}, \ldots, \bar{m}^{s}$ on the subspaces $E_{p}^{u}, \ldots, E_{p}^{s}$. On these linear subspaces, and on all affine subspaces of $T_{p} M$ parallel to them, we also have the Riemann measures $m_{p}^{u}, \ldots, m_{p}^{s}$ induced by the inner product. Again, one can view the latter measures as the linear parts of the former measures. 
Lemma 8.1. The volume of a small tube is approximately the volume of its base times the volume of its fiber.

$$
\frac{m(T)}{m^{c u}(B) m^{s}\left(W^{s}(p, r)\right)} \approx 1 .
$$

More precisely, for any $\epsilon_{0}>0$ there is a $\delta>0$ such that if $T$ is a solid tube with base $B \subset W^{c u}(p, \delta)$ and stable radius $r<\delta$ then

$$
\left|\frac{m(T)}{m^{c u}(B) m^{s}\left(W^{s}(p, r)\right)}-1\right|<\epsilon_{0} .
$$

Corresponding assertions are true for center unstable tubes and center stable tubes.

Proof. According to (25), when $T$ is small,

$$
\frac{m_{p}(\bar{T})}{m(T)} \approx 1 \quad \frac{m_{p}^{c u}(\bar{B})}{m^{c u}(B)} \approx 1 \quad \frac{m_{p}^{s}\left(\bar{W}^{s}(p, r)\right)}{m^{s}\left(W^{s}(p, r)\right)} \approx 1 .
$$

It is therefore fair in the proof of (26) to replace the tube, its base, its fiber, and the various measures by corresponding objects in $T_{p} M$.

Since $e_{p}$ is adapted to the invariant foliations of $f$, the base $\bar{B}$ of $\bar{T}$ lies in $E_{p}^{c u}$. Due to (25), given $\epsilon>0$, it actually lies in $E_{p}^{c u}((1+\epsilon) \delta)$ when $T$ is small.

The main stable fiber of $\bar{T}$ is $e_{p}^{-1}\left(W^{s}(p, r)\right)=\bar{W}^{s}(p, r)$. Since $e_{p}$ is adapted to the invariant foliations of $f, \bar{W}^{s}(p, r)$ lies in $E_{p}^{s}$. By (25), when $T$ is small it is bracketted between round stable discs

$$
\left.E_{p}^{s}((1-\epsilon / 2) r) \subset \bar{W}^{s}(p, r)\right) \subset E_{p}^{s}((1+\epsilon / 2) r) .
$$

Let $\pi_{p}^{s}: T_{p} M \rightarrow E_{p}^{s}$ be the projection with kernel $E_{p}^{c u}$. Since, when $T$ is small, the stable fibers of $\bar{T}$ uniformly $C^{1}$ approximate the main stable fiber, their $\pi_{p}^{s}$-projections into $E_{p}^{s}$ are bracketted between discs with slightly relaxed radii, say $Y_{ \pm}=E_{p}^{s}((1 \pm \epsilon) r)$. In particular,

$$
Y_{-} \subset \bar{W}^{s}(p, r) \subset Y_{+} .
$$

It also follows that $\bar{T}$ is bracketted between $T_{ \pm}$,

$$
T_{-} \subset \bar{T} \subset T_{+},
$$

where

$$
T_{ \pm}=\left\{z \in \bar{W}^{s}(x, 2 r): x \in \bar{B} \text { and } \pi_{p}^{s}(z) \in Y_{ \pm}\right\} .
$$

See Fig. 15. The set $T_{ \pm}$is sliced perfectly by the affine subspaces $E_{p}^{c u} \times y$, $y \in Y_{ \pm}$. In fact the intersections of $T_{ \pm}$with these affine subspaces are the holonomy images of the base, $\bar{h}_{y}(\bar{B})$. The stable foliation is absolutely 


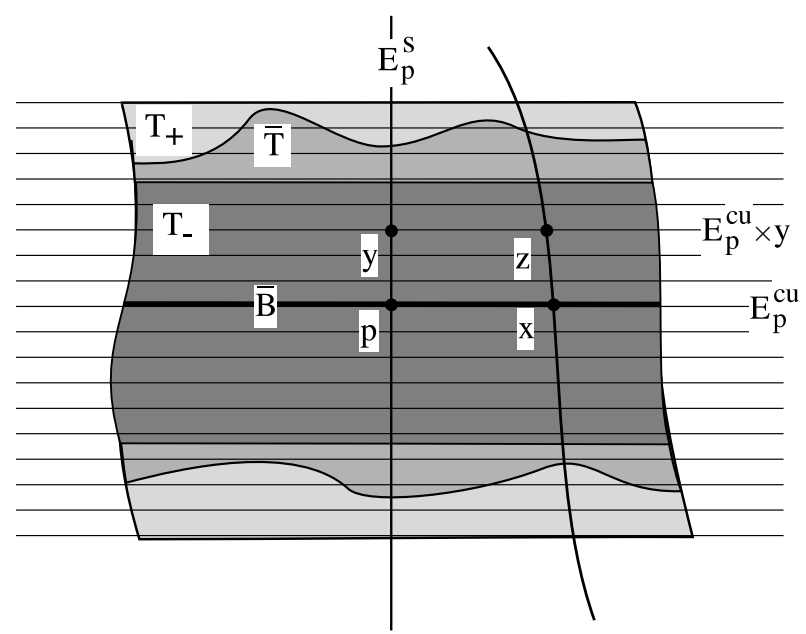

Fig. 15. Why Fubini's Theorem applies to $T_{ \pm}$but not directly to $\bar{T}$. Note that $\bar{h}_{y}: \bar{B} \rightarrow$ $E_{p}^{c u} \times y$ is the stable holonomy map that sends $x$ to $z$

continuous, and so is its lift to $T_{p} M$. The Radon Nikodym derivative of its transverse holonomy, $R N\left(\bar{h}_{y}\right)$, is continuous. When $y=0, \bar{h}_{y}$ is the identity and the Radon Nikodym derivative is 1 . By continuity, when $y$ is small, $R N\left(\bar{h}_{y}\right) \approx 1$. Thus, by Fubini's Theorem

$$
\frac{m_{p}\left(T_{ \pm}\right)}{m_{p}^{c u}(\bar{B}) m_{p}^{s}\left(Y_{ \pm}\right)}=\frac{1}{m_{p}^{s}\left(Y_{ \pm}\right)} \int_{y \in Y_{ \pm}} \frac{m_{p}^{c u}\left(\bar{h}_{y}(\bar{B})\right)}{m_{p}^{c u}(\bar{B})} d m_{p}^{s} \approx 1,
$$

if $T$ is small. Since $Y_{ \pm}$are round discs,

$$
\frac{m_{p}^{s}\left(Y_{+}\right)}{m_{p}^{s}\left(Y_{-}\right)}=\left(\frac{1+\epsilon}{1-\epsilon}\right)^{s} \approx 1
$$

Since $T_{ \pm}$bracket $\bar{T}$ and $Y_{ \pm}$bracket $\bar{W}^{s}(p, r)$,

$$
\frac{m_{p}\left(T_{-}\right)}{m_{p}^{c u}(\bar{B}) m_{p}^{s}\left(Y_{+}\right)} \leq \frac{m_{p}(\bar{T})}{m_{p}^{c u}(\bar{B}) m_{p}^{s}\left(\bar{W}^{s}(p, r)\right)} \leq \frac{m_{p}\left(T_{+}\right)}{m_{p}^{c u}(\bar{B}) m_{p}^{s}\left(Y_{-}\right)} .
$$

Hence, (27), (28), (29) imply (26).

Next we give an estimate to control volume of a small set along an orbit of our $C^{2}$ diffeomorphism $f: M \rightarrow M$. We assume that $E$ is a $\theta$-Hölder continuous, integrable, $T f$-invariant $k$-dimensional subbundle of $T M, \theta>0$. We denote by $U$ a small $k$-dimensional disc tangent to $E$ with $p \in U$, and by $m_{E}$ the Riemann measure on such a disc. The restriction of $T f$ to $E$ is $T^{E} f$. We have in mind the example that $U$ is a small center disc, a small center stable disc, or a small solid neighborhood. Then $E=E^{c}, E^{c s}, T M$ respectively. 
Theorem 8.2. The restriction of a high iterate of $f$ to a small enough disc tangent to $E$ is approximately measure theoretically conformal in the following sense. Fix a constant $\kappa<1$. If the disc $U$ is so small that for $i=0, \ldots, n$,

$$
\operatorname{diam} f^{i} U \leq \kappa^{n},
$$

then

$$
\frac{m_{E}\left(f^{i} U\right)}{\left|\operatorname{det} T_{p}^{E} f^{i}\right| m_{E}(U)} \approx 1
$$

Proof. $m_{E}\left(f^{i} U\right)$ is the integral of the absolute determinant of $T^{E} f^{i}$ over $U$. Thus,

$$
\frac{m_{E}\left(f^{i} U\right)}{\left|\operatorname{det} T_{p}^{E} f^{i}\right| m_{E}(U)}=\frac{1}{m_{E}(U)} \int_{x \in U} \prod_{j=0}^{i-1} \frac{\operatorname{det} T_{f^{j} x}^{E} f}{\operatorname{det} T_{f^{j} p}^{E} f} d m_{E},
$$

which, since $E$ is $\theta$-Hölder and $f$ is $C^{2}$, is the average value on $U$ of a product $\prod\left(1+\left(\epsilon_{j}(x)\right)^{\theta}\right)$ where

$$
\left|\epsilon_{j}(x)\right| \leq C \operatorname{diam} f^{j} U \leq C \kappa^{n} .
$$

Because $n \kappa^{n \theta} \rightarrow 0$ as $n \rightarrow \infty$, the product approximates 1 .

We have chosen the juliennes so that they remain exponentially small under $n$ iterates of $f$. According to Theorem 8.2, then, the effect of $f^{i}$, $|i| \leq n$, on the fibers of the juliennes is approximately measure theoretically conformal. Under $f^{i}$ the fiber measures get multiplied approximately by the appropriate determinant. See (30).

Theorem 8.3. Fix an integer $\ell \geq 1$. (Eventually, $\ell$ is a rank disruption bound from Theorems 6.1, 7.1, or 7.2.) There exists a constant $c=c(\ell)>0$ such that for all $p \in M$ and all large $n$,

$$
c \leq \frac{m^{c s}\left(J_{n+\ell}^{c s}(p)\right)}{m^{c s}\left(J_{n}^{c s}(p)\right)} \leq 1 .
$$

The corresponding assertions hold for center unstable juliennes and solid juliennes.

Proof. The upper bound of 1 is trivial, since juliennes nest down to $p$ as $n \rightarrow \infty$.

We first assume $\ell=1$. Since the center cores $D_{n}, D_{n+1}$ of $J_{n}^{c s}(p)$, $J_{n+1}^{c s}(p)$ are round discs of known radii $\sigma^{n}, \sigma^{n+1}$, there is a constant $c_{1}$ such that

$$
0<c_{1} \leq \frac{m^{c}\left(D_{n+1}\right)}{m^{c}\left(D_{n}\right)} .
$$


Let $B_{n}=f^{-n}\left(D_{n}\right)$ and $B_{n+1}=f^{-n}\left(D_{n+1}\right)$. Clearly, $B_{n+1} \subset B_{n}$ and they are exponentially small. According to Theorem 8.2, since the ratio of the center measures of $D_{n}, D_{n+1}$ is uniformly controlled, so is the ratio between the center measures of their iterates $B_{n}, B_{n+1}$. In fact, nearly the same control constants can be used.

Now consider the stable fibers $Y_{n}(x), Y_{n+1}(x)$ of the pre-juliennes $f^{-n}\left(J_{n}^{c s}\right), f^{-n}\left(J_{n+1}^{c s}\right)$ at $x \in B_{n}$. By Proposition 5.1, the former is approximately equal to a round disc,

$$
Y_{n}(x) \approx W^{s}\left(x, \tau^{n}\right) .
$$

The latter is bracketted between round discs,

$$
W^{s}\left(x, \mu \tau^{n+1}\right) \subset Y_{n+1}(x) \subset W^{s}\left(x, v \tau^{n+1}\right) .
$$

Thus, for some constants $c_{2}$, we have

$$
0<c_{2} \leq \frac{m^{s}\left(Y_{n+1}(x)\right)}{m^{s}\left(Y_{n}(x)\right)} .
$$

Applying Lemma 8.1 at the point $f^{-n}(p)$ with $B=B_{n}$, gives a comparison

$$
0<c_{3} \leq \frac{m^{c s}\left(f^{-n}\left(J_{n+1}^{c s}(p)\right)\right)}{m^{c s}\left(f^{-n}\left(J_{n}^{c s}(p)\right)\right)} .
$$

Iterating by $f^{n}$ and applying Theorem 8.2 again, we see that the center stable juliennes $J_{n}^{c s}(p), J_{n+1}^{c s}(p)$ enjoy the same comparability of measure,

$$
0<c_{4} \leq \frac{m^{c s}\left(J_{n+1}^{c s}(p)\right)}{m^{c s}\left(J_{n}^{c s}(p)\right)}
$$

which completes the proof when $\ell=1$. For general $\ell$ the choice $c=c_{4}^{\ell}$ give

$$
0<c \leq \frac{m^{c s}\left(J_{n+\ell}^{c s}(p)\right)}{m^{c s}\left(J_{n}^{c s}(p)\right)} .
$$

Measure comparability for center unstable juliennes has a symmetric proof.

Finally consider solid juliennes $J_{n}(p)$ and $J_{n+\ell}(p)$. According to Theorem 7.4 there is a solid tube

$$
T_{n+\ell} \subset f^{-(n+\ell)}\left(J_{n+\ell}(p)\right),
$$

which has base $f^{-(n+\ell)}\left(J_{n+\ell}^{c u}(p)\right)$ and stable discs of radius $(\mu \tau)^{L} \tau^{n+\ell}$. The $f^{\ell}$-image of $T_{n+\ell}$ contains a solid tube $T_{n}^{*}$ with base $B_{n}^{*}=f^{-n}\left(J_{n+\ell}^{c u}(p)\right)$ and stable fiber of radius $(\mu \tau)^{\ell+L} \tau^{n}$. 
Likewise there is a solid tube $T_{n}^{\prime}$ with

$$
f^{-n}\left(J_{n}(p)\right) \subset T_{n}^{\prime},
$$

which has base $B_{n}^{\prime}=f^{-n}\left(J_{n}^{c u}(p)\right)$ and stable discs of radius $(\mu \tau)^{-L} \tau^{n}$. Thus

$$
T_{n}^{*} \subset f^{\ell}\left(T_{n+\ell}\right) \subset f^{-n}\left(J_{n+\ell}(p)\right) \subset f^{-n}\left(J_{n}(p)\right) \subset T_{n}^{\prime} .
$$

We claim that $T_{n}^{*}$ and $T_{n}^{\prime}$ have comparable measures. Both are solid tubes, and their bases nest

$$
B_{n}^{*} \subset B_{n}^{\prime} .
$$

In fact $f^{n}\left(B_{n}^{*}\right)=J_{n+\ell}^{c u}(p)$ while $f^{n}\left(B_{n}^{\prime}\right)=J_{n}^{c u}(p)$. We have already established measure comparability for these center unstable juliennes at $p$. By Theorem 8.2 their measures remain comparable under $f^{-n}$. That is, the bases $B_{n}^{*}$ and $B_{n}^{\prime}$ have compaarble measures. Since the radii of their stable discs have ratio no less than $(\mu \tau)^{\ell+2 L}$, the measures of $T_{n}^{*}$ and $T_{n}^{\prime}$ are comparable, and the same is true for the sets they bracket, namely $f^{-n}\left(J_{n+\ell}(p)\right)$ and $f^{-n}\left(J_{n}(p)\right)$. Applying Theorem 8.2 again gives measure comparability of the solid juliennes $J_{n+\ell}(p)$ and $J_{n}(p)$.

Theorem 8.4. The Vitali bases $\mathscr{J}, \mathscr{J}^{c u}$, and $\mathscr{J}^{c s}$ are volumetrically engulfing, and hence are density bases.

Proof. The assertion for $\mathscr{J}$ means that if $S \subset M$ is measurable with respect to the Riemann measure $m$ on $M$ then almost every point of $S$ is a density point with respect to $\mathscr{J}$. In Theorem 7.2 we showed that a solid julienne $J_{n}$ is engulfed by $J_{n-L}^{\prime}$ if $J_{n}$ meets $J_{n}^{\prime}$. In Theorem 8.3 we showed that there is a uniform bound on the volume ratio between engulfer and engufee. Thus, Theorem 3.1 applies and $\mathscr{T}$ is a density basis.

The assertion for $\mathscr{J}^{c u}$ means that if $S \subset W^{c u}(p)$ is measurable with respect to Riemann leaf measure $m^{c u}$ then almost every point of $S$ is a density point with respect to $\mathscr{J}^{c u}$. The proof is similar.

Theorem 8.5. The stable and unstable holonomy maps are julienne quasiconformal, and hence any unstable holonomy map preserves all $\mathscr{J}^{c s}$ density points, while a stable holonomy map preserves all $\mathscr{J}^{c u}$ density points.

Proof. According to Theorems 6.1 and 8.3, if $q \in W^{u}(p, 1)$ then the unstable holonomy map $h: W_{\mathrm{loc}}^{c s}(p) \rightarrow W_{\mathrm{loc}}^{c s}(q)$ satisfies

$$
J_{n+k}^{c s}(q) \subset h\left(J_{n}^{c s}(p)\right) \subset J_{n-k}^{c s}(q),
$$

and the measure ratio between $J_{n+k}^{c s}(q)$ and $J_{n-k}^{c s}(q)$ is bounded away from zero. That is, $h$ is julienne quasi-conformal. According to Proposition 2.1, $h$ preserves all $\mathscr{J}^{c s}$ density points. The dual statements hold for a stable holonomy map. 
Next, we relate density points of a set to density points of the stable projection of that set onto a local center unstable manifold.

Theorem 8.6. Assume that $A$ is measurable and s-saturated. The point $p$ is a density point of $A$ with respect to the solid julienne basis $\mathscr{J}$ if and only if $p$ is a density point of $A \cap W^{c u}(p)$ with respect to the center unstable julienne density basis $\mathscr{J}^{c u}$.

Proof. Suppose that $p$ is a density point of $A$ with respect to $\mathscr{J}$. Then

$$
\left[A: J_{n}(p)\right] \rightarrow 1
$$

as $n \rightarrow \infty$. According to Theorem $8.2, f^{-n}(A)$ is also highly concentrated in the pre-julienne $f^{-n}\left(J_{n}(p)\right)$,

$$
\left[f^{-n}(A): f^{-n}\left(J_{n}(p)\right)\right] \rightarrow 1 .
$$

By Theorem 7.4 there is a tube $T_{n} \subset f^{-n}\left(J_{n}(p)\right)$ whose volume ratio in the pre-julienne is bounded away from zero. Hence

$$
\left[f^{-n}(A): T_{n}\right] \rightarrow 1 .
$$

The base of the tube is $f^{-n}\left(J_{n+L}^{c u}\right)$ and its stable radius is $r_{n}$. Since $A$ is $s$-saturated, so is $f^{-n}(A)$. Thus $f^{-n}(A)$ consists of whole stable manifolds, and its intersection with the tube $T_{n}$ consists of whole stable discs of equal radius $r_{n}$. We apply Lemma 8.1 to two tubes: the tube $T_{n}$ and the tube $T_{n} \cap f^{-n}(A)$. This gives

$$
\frac{m\left(T_{n} \cap f^{-n}(A)\right)}{m^{c u}\left(f^{-n}\left(J_{n+L}^{c u}\right) \cap f^{-n}(A)\right) m^{s}\left(W^{s}\left(f^{-n}(p), r_{n}\right)\right)} \approx 1
$$

while the volume of $T_{n}$ itself satisfies

$$
\frac{m\left(T_{n}\right)}{m^{c u}\left(f^{-n}\left(J_{n+L}^{c u}\right)\right) m^{s}\left(W^{s}\left(f^{-n}(p), r_{n}\right)\right)} \approx 1 .
$$

Hence, the concentration of $f^{-n}(A)$ in the base $f^{-n}\left(J_{n+L}^{c u}\right)$ must tend to 1 . Applying $f^{n}$ to this configuration and using Theorem 8.2 again, we see that the concentration of $A$ in the center unstable julienne $J_{n+L}^{c u}$ also tends to 1 . Since $L$ is fixed, this shows that $p$ is a density point of $A \cap W^{c u}(p)$ with respect to $\mathscr{J}^{c u}$.

The converse is proved similarly, using the large tube $T_{n}^{\prime}$ from Theorem 7.4.

We combine Theorems 8.5 and 8.6 as follows. 
Theorem 8.7. If $A \subset M$ is measurable and essentially $u$-saturated then its set of $\mathscr{J}$ density points consists of whole unstable manifolds.

Proof. A set is essentially $u$-saturated if it differs from a $u$-saturated set by a zero set. Alteration by a zero set has no effect on the set of (solid) density points, so it is fair to assume that $A$ is $u$-saturated. Let $p$ be a $\mathscr{J}$ density point and let $q$ be a point in $W^{u}(p)$. We claim that $q$ is a $\mathscr{J}$ density point of $A$.

Assume at first that $q$ lies in the local unstable manifold of $p$. By Theorem 8.6, $p$ is a density point of $A \cap W^{c s}(p)$ with respect to $\mathscr{J}^{c s}$. By Theorem 8.5 and the fact that $A$ is $u$-saturated, $q$ is a $\mathscr{J}^{c s}$ density point of $A \cap W^{c s}(q)$. By Theorem 8.6, $q$ is a $\mathscr{J}$ density point of $A$. Thus, the set $P$ of $\mathscr{J}$ density points consists of whole local unstable manifolds. Because (global) unstable manifolds are connected and consist locally of local unstable manifolds, the set $P$ of density points actually consists of whole unstable manifolds.

\section{Proof of Theorem A}

The proof of Theorem A follows the same pattern as in [2,12,21]. We suppose that $f$ is not ergodic and, using the Birkhoff Ergodic Theorem, we find a set $A_{0}$ such that $A_{0}$ is $f$-invariant, has intermediate measure, and is essentially saturated by stable and unstable manifolds. Let $A$ be the set of $\mathscr{J}$ density points of $A_{0}$ and let $B$ be the set of $\mathscr{J}$ density points of $M \backslash A_{0}$. According to Theorem 8.4, almost every point of $A_{0}$ is a $\mathscr{J}$ density point, so $A$ has positive measure. Likewise the set $B$ has positive measure. The set $P$ of all $u s$-paths originating at points of $A$ is, by definition, $u s$-saturated and it contains $A$, so it forms a $u s$-saturated set of positive measure. Essential accessibility implies that $P$ has full measure, and therefore it meets $B$. The upshot is that there are points $p \in A, q \in B$ and a $u s$-path from $p$ to $q$. Since $p$ is a $\mathscr{J}$ density point of $A_{0}$, Theorem 8.7 implies that the entire first leg of the path consists of $\mathscr{J}$ density points of $A_{0}$. The same applies to the second leg, and so forth. We conclude that $q$ is also a $\mathscr{J}$ density point of $A_{0}$ and this is incompatible with it also being a $\mathscr{J}$ density point of $M \backslash A_{0}$.

\section{Affine diffeomorphisms}

In this section we prove Theorems B, C, and Corollaries b, c from Sect. 1. For readers unfamiliar with Lie groups, we include some standard material, see also [21] where some of this is worked out for the case of left translation. We use the standard notation from differential topology that $T_{x} M$ is the tangent space to $M$ at $x$ and that $T_{x} f$ is the tangent to a map $f$ at $x$. 
Recall what we assumed in Sect. 1.

(a) $G$ is a connected Lie group and $B \subset G$ is a proper, closed subgroup.

(b) $G / B$ is compact.

(c) Haar measure projects to a finite measure $m$ on $G / B$, invariant under left translation, $x B \mapsto g x B$

(d) $f: G / B \rightarrow G / B$ is an affine diffeomorphism. That is, it is part of a commutative diagram

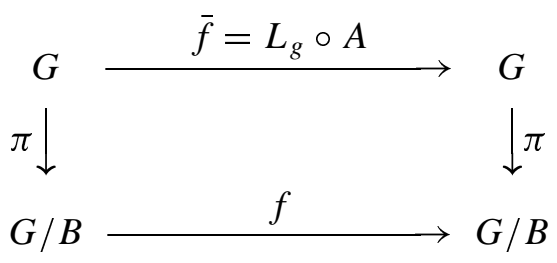

where $A$ is an automorphism of $G, L_{g}$ is left multiplication by some fixed element $g \in G$, and, to make the projection well defined, $A(B)=B$.

Further, $\bar{f}$ induces a Lie algebra automorphism defined by

$$
\mathfrak{a}(\bar{f})=A d(g) \circ T_{e} A=T_{g} R_{g^{-1}} \circ T_{e} L_{g} \circ T_{e} A .
$$

Given a linear subspace $\bar{E}$ of the Lie algebra $\mathfrak{g}=T_{e} G$, we extend it to a right invariant subbundle $R(\bar{E}) \subset T G$ whose fiber at $x \in G$ is

$$
\bar{E}_{x}=T_{e} R_{x}(\bar{E}) .
$$

Right invariance of $R(\bar{E})$ means that for all $x, y \in G, T_{x} R_{y}$ carries $\bar{E}_{x}$ to $\bar{E}_{x y}$.

Proposition 10.1. $\mathfrak{a}(\bar{f})$-invariance of $\bar{E}$ is equivalent to $T \bar{f}$-invariance of $R(\bar{E})$.

Proof. Observe first that

$$
T_{e} \bar{f}=T_{e} R_{g} \circ \mathfrak{a}(\bar{f}) .
$$

For $\bar{f}(x)=g A(x)=L_{g} \circ A(x)$ implies that

$$
T_{e} R_{g} \circ \mathfrak{a}(\bar{f})=T_{e} R_{g} \circ T_{g} R_{g^{-1}} \circ T_{e} L_{g} \circ T_{e} A=T_{e} L g \circ T_{e} A,
$$

which is $T_{e} \bar{f}$.

From (31) and right invariance of $R(\bar{E})$ we infer that $\bar{E}$ is $\mathfrak{a}(\bar{f})$-invariant if and only if $T_{e} \bar{f}$ sends $\bar{E}=\bar{E}_{e}$ to $\bar{E}_{g}$. It remains to show that this $T \bar{f}$ invariance of $R(\bar{E})$ from $e$ to $\bar{f}(e)=g$ implies $T \bar{f}$-invariance at the general point $y \in G$. 
Commutativity of the diagram

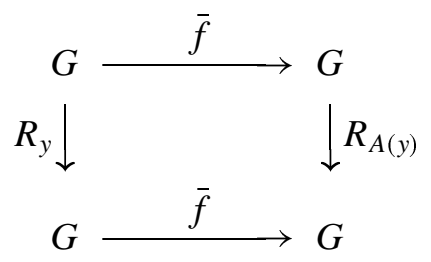

is simple to check: if $x \in G$ then

$$
R_{A(y)} \circ \bar{f}(x)=g A(x) A(y)=g A(x y)=\bar{f}(x y)=\bar{f}\left(R_{y}(x)\right) .
$$

Taking the tangent maps of the diagram at $x=e$ gives

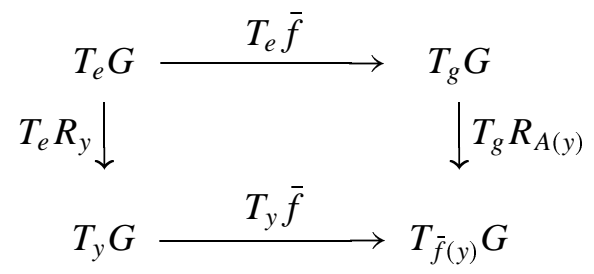

Hence

$$
T_{y} \bar{f}=T_{g} R_{A(y)} \circ T_{e} \bar{f} \circ\left(T_{e} R_{y}\right)^{-1} .
$$

Since $R(\bar{E})$ is $T R$-invariant, it is also $(T R)^{-1}$-invariant, and $T \bar{f}$-invariance of $R(\bar{E})$ from $e$ to $\bar{f}(e)$ propagates to $T \bar{f}$-invariance everywhere.

Because $B$ acts on the right, the bundle $R(\bar{E})$ projects naturally to a quotient bundle, $T \pi(R(\bar{E}))=R(E) \subset T(G / B)$, where $E=T \pi(\bar{E})$,

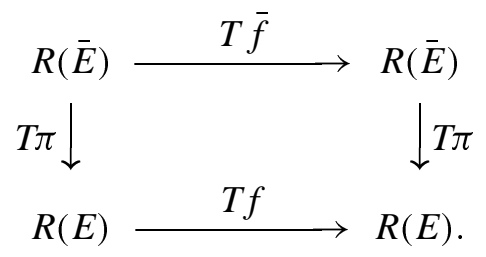

The fiber of $R(E)$ at $x B \in G / B$ is $T_{x} \pi\left(\bar{E}_{x}\right)$, which is isomorphic to $\bar{E}_{x} /\left(T_{x}(x B) \cap \bar{E}_{x}\right)$. Here, we think of $x B$ both as a subset of $G$ and as a point in $G / B$.

Corollary 10.2. $\mathfrak{a}(\bar{f})$-invariance of $\bar{E}$ implies $T f$-invariance of $R(E)$.

Proof. From commutativity of the diagrams this is clear.

Proposition 10.3. Let $\mathfrak{g}_{\rho}$ be the sum of the generalized eigenspaces of $\mathfrak{a}(\bar{f})$ whose eigenvalues have modulus $\leq \rho$ where $0<\rho \leq 1$. Then $\mathfrak{g}_{\rho}$ is a Lie subalgebra of $\mathfrak{g}$, not merely a linear subspace of $T_{e} G$. 
Proof. The assertion is valid not only for $\mathfrak{a}(\bar{f})$, but for any Lie algebra automorphism $\mathfrak{a}: \mathfrak{g} \rightarrow \mathfrak{g}$ of a complex, finite dimensional Lie algebra, and is a consequence of the following general fact: the factors $S$ and $M$ in the Jordan Decomposition $\mathfrak{a}=S M$, are automatically Lie algebra automorphisms of $\mathfrak{g}$, not merely linear transformations $T_{e} G \rightarrow T_{e} G$. As usual $S$ is semi-simple and $M=I+N$ with $N$ nilpotent. See Chapt. I.4 of Borel's book about linear algebraic groups [3] where this is discussed. Note that the automorphism group of $\mathfrak{g}$ is a linear algebraic group.

Suppose first that $\mathfrak{a}$ itself is semi-simple, $\mathfrak{a}=S$. Then there is a complete eigenbasis $\left\{v_{1}, \ldots, v_{n}\right\}$ corresponding to eigenvalues $\left\{\lambda_{1}, \ldots, \lambda_{n}\right\}$. Since $\mathfrak{a}$ is a Lie algebra automorphism,

$$
S\left[v_{i}, v_{j}\right]=\left[S v_{i}, S v_{j}\right]=\lambda_{i} \lambda_{j}\left[v_{i}, v_{j}\right],
$$

and $\left[v_{i}, v_{j}\right]$ is seen to be either the zero vector or an $S$-eigenvector with eigenvalue $\lambda_{i} \lambda_{j}$. In particular, $\mathfrak{g}_{\rho}$ is closed under Lie bracket since $\left|\lambda_{i}\right|,\left|\lambda_{j}\right| \leq$ $\rho \leq 1$ implies that $\left|\lambda_{i} \lambda_{j}\right| \leq \rho^{2} \leq \rho$.

Next assume that $\mathfrak{a}=S M$ with $S$ semi-simple, $M=I+N$, and $N \neq 0$ nilpotent. Since $M S=S M$, the $S$-eigenvalues are identical with the $\mathfrak{a}$-eigenvalues and the $S$-eigenspaces are identical with the generalized $\mathfrak{a}$-eigenspaces. The Lie bracket operation is defined independently from $\mathfrak{a}, S, M$, so the conclusions for $S$ hold also for $\mathfrak{a}$.

The Lie algebra automorphism $\mathfrak{a}(\bar{f})$ in the statement of the proposition is real, not complex, but since eigenvalues, generalized eigenspaces, and brackets act naturally under complexification, the complex case implies the real one.

As in Sect. 1 we split $\mathfrak{g}$ and $\mathfrak{a}(\bar{f})$ according to the generalized eigenspaces of with eigenvalues of modulus $>1,=1$, and $<1$ respectively,

$$
\begin{aligned}
& \mathfrak{a}(\bar{f})=\mathfrak{a}^{u}(\bar{f}) \oplus \mathfrak{a}^{c}(\bar{f}) \oplus \mathfrak{a}^{s}(\bar{f}) \\
& \mathfrak{g}=\mathfrak{g}^{u} \oplus \mathfrak{g}^{c} \oplus \mathfrak{g}^{s} .
\end{aligned}
$$

By Proposition 10.3 these are Lie subalgebras of $\mathfrak{g}$. We extend them to right invariant bundles $\bar{E}^{u}, \bar{E}^{c}, \bar{E}^{s}$ over $G$. According to Proposition 10.1, these bundles are $T \bar{f}$-invariant. By Corollary 10.2 their projections to $G / B, E^{u}$, $E^{c}$, and $E^{s}$ are $T f$-invariant,

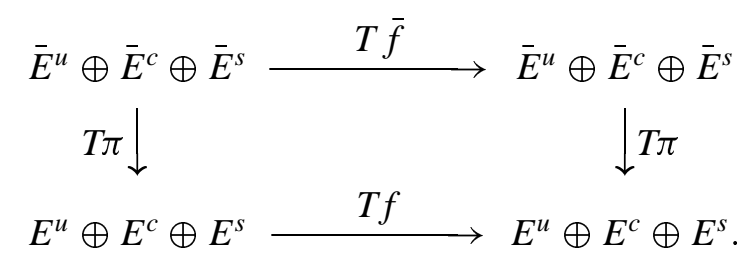

A priori, some of these bundles may be zero. 
Proposition 10.4. There is a Riemann structure on $T(G / B)$ adapted to $T f$ with respect to which $T f$ expands $E^{u}$ sharply, $T f$ is nearly isometric on $E^{c}$, and $T f$ contracts $E^{s}$ sharply.

Proof. Choose $\rho, 0<\rho<1$, such that the moduli of the eigenvalues of $\left(\mathfrak{a}^{u}(\bar{f})\right)^{-1}$ and $\mathfrak{a}^{s}(\bar{f})$ lie in the interval $(0, \rho)$. For any $\epsilon>0$ there is an inner product on $T_{e} G$ which is adapted to $\mathfrak{a}(\bar{f})$ in the sense that the norms of $\left(\mathfrak{a}^{u}(\bar{f})\right)^{-1}$ and $\mathfrak{a}^{s}(\bar{f})$ are $<\rho$, while the norms of $\left(\mathfrak{a}^{c}(\bar{f})\right)^{-1}$ and $\mathfrak{a}^{c}(\bar{f})$ are $<1+\epsilon$. Right translate this inner product to a right invariant Riemann structure on $T G$. Right translations become isometries. By (31) and the proof of Proposition 10.1, $T_{e} \bar{f}$ is isometric to $T_{x} \bar{f}$ for all $x \in G$. Hence, the Riemann structure is adapted to $T \bar{f}$. Vectors in $\bar{E}^{u}$ are expanded more sharply than the factor $1 / \rho$, vectors in $\bar{E}^{s}$ are contracted more sharply than the factor $\rho$, and vectors in $\bar{E}^{c}$ are affected isometrically, up to factors $1-\epsilon$ and $1+\epsilon$.

Since $B$ acts on the right, and since the $T \bar{f}$-adapted Riemann structure is right invariant, it projects by $T \pi: T G \rightarrow T(G / B)$ to a Riemann structure on $T(G / B)$. Under quotients, norms become no larger. Hence, $\left\|T^{s} f\right\| \leq$ $\left\|T^{s} \bar{f}\right\|<\rho$. Similarly, $\left\|T^{c s} f\right\| \leq\left\|T^{c s} \bar{f}\right\|$, and if the bundles are non-zero so the inverses exist, $\left\|T^{u} f^{-1}\right\| \leq\left\|T^{u} \bar{f}^{-1}\right\|$ and $\left\|T^{c u} f^{-1}\right\| \leq\left\|T^{c u} \bar{f}^{-1}\right\|$. Hence, $T^{u} f$ expands by at least the factor $1 / \rho, T^{s} f$ contracts by at most the factor $\rho$, and since $E^{c}=E^{c u} \cap E^{c s}, T^{c} f$ is an isometry up to a factor between $1-\epsilon$ and $1+\epsilon$.

Recall that $\mathfrak{h}$ is the hyperbolic Lie subalgebra of $\bar{f}$ and $\mathfrak{b}$ is the Lie algebra of $B$. Both are subalgebras of $\mathfrak{g}$.

Corollary 10.5. The affine diffeomorphism $f$ is partially hyperbolic if and only if $\mathfrak{h} \not \subset \mathfrak{b}$.

Proof. We prove the equivalence of the opposites. If $\mathfrak{h} \subset \mathfrak{b}$ then $T(G / B)=$ $E^{c}$ and $f$ is completely central; it is not partially hyperbolic. On the other hand, if $f$ is not partially hyperbolic then at least one of $E^{u}, E^{s}$ is zero, say

$$
T(G / B)=E^{c} \oplus E^{s} .
$$

Since $f$ preserves the projected Haar measure, the determinant of $T f$ is \pm 1 everywhere, which implies that $E^{s}=0$ also. Thus $\bar{E}^{u}, \bar{E}^{s} \subset \mathfrak{b}$, and $\mathfrak{h} \subset \mathfrak{b}$.

Proposition 10.6. The right invariant bundles $\bar{E}^{u}, \bar{E}^{c u}, \bar{E}^{c}, \bar{E}^{c s}, \bar{E}^{s}$ and their projections to $T(G / B)$ are tangent to smooth dynamically coherent foliations; i.e., $\bar{f}$ and $f$ are dynamically coherent. 
Proof. Since $\mathfrak{g}^{u}, \mathfrak{g}^{c u}, \mathfrak{g}^{c}, \mathfrak{g}^{c s}, \mathfrak{g}^{s}$ are Lie subalgebras of $\mathfrak{g}$ they are tangent to unique connected, locally closed subgroups, $G^{u}, G^{c u}, G^{c}, G^{c s}, G^{s}$ respectively. The orbits $G^{u} x$ of the left $G^{u}$-action are integral manifolds of the right invariant subbundle $\bar{E}^{u}$. For if we consider the general point $h x \in G^{u} x$ then the right multiplication diffeomorphism $R_{h x}: G \rightarrow G$ sends $G^{u}$ onto $G^{u} x$, sends $e$ to $h x$, and hence

$$
T_{h x}\left(G^{u} x\right)=\left(T_{e} R_{h x}\right)\left(T_{e} G^{u}\right)=\left(T_{e} R_{h x}\right)\left(\mathfrak{g}^{u}\right)=\left(T_{e} R_{h x}\right)\left(\bar{E}_{e}^{u}\right)=\bar{E}_{h x}^{u}
$$

by right invariance of $\bar{E}^{u}$. The corresponding facts are true for the other subgroups. Also, these subgroups act on $G / B$ and their orbits integrate the projected bundles there.

Recall that the smallest Lie subalgebra of $\mathfrak{g}$ containing $\mathfrak{g}^{u}$ and $\mathfrak{g}^{s}, \mathfrak{h}$, is an ideal. It is therefore the tangent space at $e$ of a unique connected, locally closed, normal, Lie subgroup $H$ of $G$.

Proposition 10.7. An element $y \in G / B$ is us-accessible from $x \in G / B$ if and only is there is an $h \in H$ such that $y=h x$.

Proof. Suppose that $y$ is accessible from $x$. From the proof of Proposition 10.6 it follows that there are elements $u_{1}, \ldots, u_{k} \in G^{u}$ and $s_{1}, \ldots, s_{k} \in$ $G^{s}$ such that

$$
y=u_{k} s_{k} u_{k-1} s_{k-1} \ldots u_{1} s_{1} x .
$$

Since $H$ contains $G^{u}$ and $G^{s}$, the product $u_{k} \ldots s_{1}$ lies in $H$. The converse can be proved similarly since $\mathfrak{h}$ is generated by $\mathfrak{g}^{u}$ and $\mathfrak{g}^{s}$.

Proof of Theorem B. Recall that for an affine diffeomorphism $f$, Theorem B asserts

(a) Partial hyperbolicity is equivalent to $\mathfrak{h} \not \subset \mathfrak{b}$.

(b) Center bunching and dynamical coherence are automatic.

(c) Accessibility in $G / B$ is equivalent to $\mathfrak{g}=\mathfrak{b}+\mathfrak{h}$.

Corollary 10.5 gives (a); Proposition 10.4 gives center bunching; Proposition 10.6 gives dynamical coherence.

It remains to prove (c). Consider the connected Lie subgroup $H$ of $G$ that is tangent at $e$ to the hyperbolic Lie subalgebra $\mathfrak{h}$. According to Proposition 10.7, accessibility in $G / B$ is equivalent to

$$
H B=G,
$$

which, since $G$ is connected, is true if and only if $\mathfrak{h}+\mathfrak{b}=\mathfrak{g}$. 
Proof of Theorem $C$. Recall that Theorem $\mathrm{C}$ asserts the stable ergodicity of the affine diffeomorphism $f$ if $\mathfrak{g}=\mathfrak{h}+\mathfrak{b}$. According to Theorem $\mathrm{B}$, $f$ satisfies all the hypotheses of Theorem A (which implies it is stably ergodic) except one: stable us-accessibility. However, we know that the unstable and stable foliations are smooth (since they are orbits of subgroups), and, by Theorem B, they have the (a priori not stable) $u s$-accessibility property. But, as we showed in [21], smooth accessibility implies stable accessibility, and Theorem A applies: $f$ is stably ergodic.

Proof of Corollary $b$. Recall that Corollary b gives a necessary and sufficient condition for stable ergodicity of a special type of affine diffeomorphism $f$. The assumptions are:

(a) $G$ is simple.

(b) $B$ is a uniform discrete subgroup of $G$.

(c) $f=L_{g}$ is left translation by some fixed $g \in G$. (In the previous notation, this means that $A$ is the identity automorphism.)

Under these hypotheses it is asserted that

$f$ is stably ergodic if and only if $\operatorname{Ad}(g)$ has at least one eigenvalue with modulus $\neq 1$.

By Proposition 5.3 of [21], $\mathfrak{h}$ is an ideal in $\mathfrak{g}$. If $A d(g)$ has an eigenvalue with modulus $\neq 1, \mathfrak{h}$ is non-trivial. Simplicity of $G$ implies that $\mathfrak{h}=\mathfrak{g}$. Theorems $\mathrm{B}$ and $\mathrm{C}$ show that $L_{g}$ is stably ergodic among $C^{2} m$-preserving diffeomorphisms of $G / B$. For the converse, we know from [4], that stable ergodicity of $L_{g}$, even among left translations, implies that $\mathfrak{h}=\mathfrak{g}$. Hence $\mathfrak{h}$ is non-trivial and $A d(g)$ has an eigenvalue with modulus $\neq 1$.

Proof of Corollary c. Recall that Corollary c concerns the special linear group $S L(n, \mathbb{R})$. One assumes that

(a) $B$ is a uniform discrete subgroup of $G=S L(n, \mathbb{R})$.

(b) $f: G / B \rightarrow G / B$ is left multiplication by some fixed matrix $M \in G$.

The assertion is that $f$ is stably ergodic among $C^{2}$ volume preserving diffeomorphisms of $G / B$ if and only if $M$ has at least one eigenvalue with modulus $\neq 1$.

To deduce Corollary c from Corollary b we note that $S L(n, \mathbb{R})$ is simple, and as in Proposition 5.6 of [21], $A d(M)$ has an eigenvalue with modulus $\neq 1$ if and only if $M$ does.

\section{An accessibility example}

Let $f$ be a partially hyperbolic diffeomorphism of $M$ with splitting $E^{u} \oplus$ $E^{c} \oplus E^{s}$. It has the accessibility property if every pair of points $p, q \in M$ 
can be joined by a $u s$-path. If this remains true for all perturbations of $f$ then $f$ (or the pair $E^{u}, E^{s}$ ) has the stable accessibility property.

Question 1. Does accessibility imply stable accessibility?

When $E^{u}, E^{s}$ are of class $C^{1}$ the answer is "yes", a fact we made use of in the proof of Theorem $C$ in Sect. 10. In general, however, $E^{u}, E^{s}$ have only the following regularity: they are Hölder and uniquely integrable. (Unique integrability of a plane field $E$ means that $E$ is tangent to a foliation and any curve everywhere tangent to $E$ lies in a leaf of the foliation. See [23] for more discussion of this.)

In this section we give an example indicating that the answer to Question 1 may be "no". Although not definitive, it shows the limits of too naive an approach.

Let $E^{u}, E^{s} \subset T M$ be fixed subbundles. We say that a point $q \in M$ is strongly accessible from $p \in M$ if the following non-zero degree condition holds. There are continuous, uniquely integrable vector fields $X^{1}, \ldots, X^{2 k}$ such that

(a) The vector fields with odd index are subordinate to $E^{u}$, and the ones with even index are subordinate to $E^{s}$.

(b) A concatenation of the flows $\varphi^{i}=\varphi_{t}^{i}(x)$ generated by the vector fields $X^{i}$ joins $p$ to $q$. That is, for some time vector $t^{*}=\left(t_{1}^{*}, \ldots, t_{2 k}^{*}\right)$,

$$
\Phi\left(t^{*}\right)=\varphi_{t_{2 k}^{*}}^{2 k} \circ \varphi_{t_{2 k-1}^{*}}^{2 k-1} \circ \cdots \circ \varphi_{t_{1}^{*}}^{1}(p)=q .
$$

(c) For some neighborhood $N$ of $t^{*}$ in $\mathbb{R}^{2 k}$ the map $\Phi: N \rightarrow M$,

$$
\Phi: t \mapsto \Phi(t),
$$

is topologically essential. That is, there exists a disc $D \subset N$ of the same dimension as $M$, and

$$
\operatorname{Degree}\left(\left.\Phi\right|_{\partial D}, q\right) \neq 0
$$

In particular, $q \notin \Phi(\partial D)$.

It is an easy application of topological degree theory to show that strong accessibility implies stable accessibility. The converse is

Question 2. Does stable accessibility imply strong accessibility?

Proposition 11.1. Local 4-legged accessibility does not imply local 4legged stable accessibility. 
Proof. Specifically, there is a pair of uniquely integrable vector fields $X, Y$ on a 4-manifold $M$ such that for some $p \in M$, and some non-empty open set $U \subset M$, every $q \in U$ is accessible from $p$ by a 4-legged $X Y$-path,

$$
\Phi\left(t_{1}, t_{2}, t_{3}, t_{4}\right)=\varphi_{t_{4}}^{Y} \circ \varphi_{t_{3}}^{X} \circ \varphi_{t_{2}}^{Y} \circ \varphi_{t_{1}}^{X}(p)=q,
$$

where $t=\left(t_{1}, t_{2}, t_{3}, t_{4}\right)$ ranges in some open $T \subset \mathbb{R}^{4}$, but there exist small, uniquely integrable perturbations $\widetilde{X}, \widetilde{Y}$ of $X, Y$ for which $\widetilde{\Phi}(T)$ is merely a 3-dimensional subset of $U$.

The construction of $X, Y$ is local. It takes place in one coordinate chart, say with coordinates $x, y, z, w$. The point $p$ is the origin, $p=(0,0,0,0)$, and the vector field $Y$ is everywhere constant,

$$
Y=\frac{\partial}{\partial y}
$$

The time set $T$ is a neighborhood of $t^{*}=(1,1,1,1)$. On the unit cube, $X$ is also constant,

$$
X=\frac{\partial}{\partial x}
$$

Thus, the first two legs of the $X Y$-path with time vector $t^{*}$ are the unit segments along the $x$-axis and parallel to the $y$-axis,

$$
[0,1] \times 0 \times 0 \times 0 \quad \text { and } \quad 1 \times[0,1] \times 0 \times 0 .
$$

Let $\beta: \mathbb{R} \rightarrow \mathbb{R}_{+}$be a smooth function with support in [4/3,5/3], and integral 1 ,

$$
\int_{\mathbb{R}} \beta(x) d x=1 .
$$

Let $\sigma: \mathbb{R}^{3} \rightarrow \mathbb{R}_{+}$be a smooth function with compact support such that $\sigma(y, z, w)=1$ whenever $|y-1| \leq 1,|z| \leq 1$, and $|w| \leq 1$.

Let $\gamma: \mathbb{R} \rightarrow[-1,1]^{2}$ be a Peano curve with compact support $[0,2]$. That is, $\gamma$ is continuous, onto, and $\gamma(y)=(0,0)$ for $y \notin[0,2]$. Necessarily $\gamma$ is not differentiable. Write $\gamma$ in components as

$$
\gamma(y)=(\zeta(y), \omega(y)) .
$$

We then define

$$
X=\frac{\partial}{\partial x}+a \frac{\partial}{\partial z}+b \frac{\partial}{\partial w}
$$

where

$$
a=\beta(x) \sigma(y, z, w) \zeta(y) \quad \text { and } \quad b=\beta(x) \sigma(y, z, w) \omega(y) .
$$


Although $X$ is not smooth with respect to $y$, its $\partial / \partial y$ component is zero. Thus, $X$ is uniquely integrable and the $X$-flow curves lie in planes $y=$ const. Specifically, if $t=\left(t_{1}, t_{2}, t_{3}, t_{4}\right)$ and

$$
\left|t_{1}-1\right|<\frac{1}{6} \quad\left|t_{2}-1\right|<1 \quad\left|t_{3}-1\right|<\frac{1}{6}
$$

then

$$
\Phi(t)=\left(t_{1}+t_{3}, t_{2}+t_{4}, \zeta\left(t_{2}\right), \omega\left(t_{2}\right)\right) .
$$

Consider the time set $T$ defined by times $t$ satisfying (32) and

$$
\left|t_{4}-1\right|<2 \text {. }
$$

Suppose that $q=(x, y, z, w)$ satisfies

$$
|x-2|<\frac{1}{6} \quad|y-2|<1 \quad|z|<1 \quad|w|<1 .
$$

Since $\gamma$ is a Peano curve, there exists $t_{2} \in[0,2]$ such that

$$
\gamma\left(t_{2}\right)=(z, w) \text {. }
$$

We then set

$$
\begin{aligned}
& t_{1}=1 \\
& t_{3}=x-1 \\
& t_{4}=y-t_{2},
\end{aligned}
$$

which makes $t=\left(t_{1}, t_{2}, t_{3}, t_{4}\right) \in T$. Therefore $\Phi(t)$ is given by (33) and

$$
\Phi(t)=q .
$$

That is, each $q$ in the set $U$ defined by (34) is the $\Phi$ image of some $t \in T$, or to put it in terms of accessibility, each $q \in U$ is accessible from $p$.

Now let $\widetilde{X}, \widetilde{Y}$ be smooth approximations to $X, Y$ of a special type:

$$
\widetilde{X}=\frac{\partial}{\partial x}+\widetilde{a} \frac{\partial}{\partial z}+\widetilde{b} \frac{\partial}{\partial w} \quad \text { and } \quad \tilde{Y}=Y=\frac{\partial}{\partial y},
$$

where

$$
\widetilde{a}=\beta(x) \sigma(y, z, w) \widetilde{\zeta}(y) \quad \widetilde{b}=\beta(x) \sigma(y, z, w) \widetilde{\omega}(y)
$$

and $\tilde{\gamma}=(\widetilde{\zeta}, \widetilde{\omega})$ is a smooth approximation to $\gamma$. The expression (33) becomes

$$
\widetilde{\Phi}(t)=\left(t_{1}+t_{3}, t_{2}+t_{4}, \widetilde{\zeta}\left(t_{2}\right), \widetilde{\omega}\left(t_{2}\right)\right)
$$

The $\widetilde{\Phi}$ image of $T$ is bracketted as

$$
[5 / 3,7 / 3] \times[1,3] \times \tilde{\gamma}(\mathbb{R}) \subset \widetilde{\Phi}(T) \subset \mathbb{R}^{2} \times \widetilde{\gamma}(\mathbb{R}),
$$

and hence has dimension 3 . 
Besides the fact that perturbations destroy accessibility in the preceding example, one can see that although all points of $U$ are accessible from $p$, no point is strongly accessible by 4-legged paths. Increasing the number of legs in the path, well beyond the dimension of $M$, may be the way to answer Questions 1 and 2 affirmatively.

\section{References}

1. Adler, R., Kitchens, B., Shub, M. (1996) Stably ergodic skew products. Discrete and Continuous Dynamical Systems 2, 349-350, 456 (Errata)

2. Anosov, D.V. (1967) Geodesic Flows on Closed Riemann Manifolds with Negative Curvature. Proc. Stek. Inst. Math \# 90

3. Borel, A. (1991) Linear Algebraic Groups, Second Edition. New York: Springer

4. Brezin, J., Shub, M. (1997) Stable ergodicity in homogeneous spaces. Bol. Soc. Brasil. Mat. (N.S.) 28(2), 197-210

5. Brin, M.I. (1975) Topological transitivity of one class of dynamic systems and flows of frames on manifolds of negative curvature. English version. Funct. Anal. Appl. 9, 9-19

6. Brin, M.I., Ya Pesin (1974) Partially hyperbolic dynamical systems. Math. USSR Izv. 8, 177-218

7. Burns, K., Pugh, C., Wilkinson, A. (2000) Stable ergodicity and Anosov flows. Topology 39(1), 149-159

8. Burns, K., Wilkinson, A. (1999) Stable ergodicity of skew products. Ann. Sci. École Norm. Sup. (4) 32(6), 879-909

9. de Guzmán, M. (1975) Differentiation of Integrals in $R^{n}$. Lect. Notes Math. \#481. Berlin, Heidelberg, New York: Springer

10. Field, M., Parry, W. (1999) Stable ergodicity of skew extensions by compact Lie groups. Topology 38(1), 167-187

11. Gehring, F., Kelley, J. (1974) Quasi-conformal Mappings and Lebesgue Density in Discontinuous Groups and Riemann Surfaces. Ann. Math. Studies \#79, 171-179, Princeton, N.J.: Princeton University Press

12. Grayson, M., Pugh, C., Shub, M. (1994) Stably ergodic diffeomorphisms. Ann. Math. 40, 295-329

13. Herman, M.R. Théorème des tores translatés et quelques applications à la stabilité topologique des systèmes dynamiques conservatifs

14. Hirsch, M., Pugh, C., Shub, M. (1977) Invariant Manifolds. Lect. Notes Math. \#583. Berlin, Heidelberg, New York: Springer

15. Hopf, E. (1939) Statistik der geodätischen Linien in Mannigfaltigkeiten negativer Krümmung, Berichte der Mathematisch-Physikalischen Klasse der Statistischen Akademie der Wissenschaften, Leipzig 11, 261-304

16. Milnor, J. (1997) Fubini foiled: Katok's paradoxical Example in Measure Theory. Math. Intelligencer 19(2), 30-32

17. Nikodym, O. (1927) Sur la mesure des ensembles plans dont tous les points sont rectilineairement accesibles. Fund. Math. 10, 116-168

18. Parry, W., Pollicott, M. (1997) Stability of mixing for toral extensions of hyperbolic systems. Tr. Mat. Inst. Steklova 216, Din. Sist. i Smezhnye Vopr., 354-363

19. Pugh, C., Shub, M. (1972) Ergodicity of Anosov actions. Invent. math. 15, 1-23

20. Pugh, C., Shub, M. (1996) Stable ergodicity and stable accessibility. Submitted to Proceedings Hangzhou dynamics conference

21. Pugh, C., Shub, M. (1997) Stably ergodic dynamical systems and partial hyperbolicity. J. Complexity 13, 125-179

22. Pugh, C., Shub, M. (1997a) Stable ergodicity and partial hyperbolicity. In International Conference on Dynamical Systems: Montevideo 1995, a tribute to Ricardo Mane. Pitman Research Notes in Math \#362 (Ledrappier, F., et al. eds.) 182-187 
23. Pugh, C., Shub, M., Wilkinson, A. (1997) Holder Foliations. Duke Math. J. 86, 517-546

24. Shub, M. (1986) Global Stability of Dynamical Systems. New York: Springer

25. Stein, E. (1993) Harmonic Analysis. Princeton, N.J.: Princeton University Press

26. Wilkinson, A. (1995) Stable ergodicity of the time one map of a geodesic flow. Ph.D thesis. Univ. of Calif. Berkeley

27. Yoccoz, J.-C. (1992) Travaux de Herman sur les Tores Invariants. In Seminaire Bourbaki, 1991-92. Astérisque \#206, 311-345 